\title{
Auditory Prosthesis with a Penetrating Nerve Array
}

\author{
John C. Middlebrooks ${ }^{1}$ and Russell L. SNyder ${ }^{2,3}$ \\ ${ }^{1}$ Kresge Hearing Research Institute, Department of Otorhinolaryngology, University of Michigan, \\ Ann Arbor, MI 48109-0506, USA \\ ${ }^{2}$ Epstein Laboratory, Department of Otolaryngology, Head and Neck Surgery, University of California, \\ San Francisco, CA 94143-0526, USA \\ ${ }^{3}$ Department of Psychology, Utah State University, Logan, UT 84322-2810, USA
}

Received: 7 September 2006; Accepted: 15 December 2006; Online publication: 30 January 2007

\begin{abstract}
Contemporary auditory prostheses ("cochlear implants") employ arrays of stimulating electrodes implanted in the scala tympani of the cochlea. Such arrays have been implanted in some 100,000 profoundly or severely deaf people worldwide and arguably are the most successful of present-day neural prostheses. Nevertheless, most implant users show poor understanding of speech in noisy backgrounds, poor pitch recognition, and poor spatial hearing, even when using bilateral implants. Many of these limitations can be attributed to the remote location of stimulating electrodes relative to excitable cochlear neural elements. That is, a scala tympani electrode array lies within a bony compartment filled with electrically conductive fluid. Moreover, scala tympani arrays typically do not extend to the apical turn of the cochlea in which low frequencies are represented. In the present study, we have tested in an animal model an alternative to the conventional cochlear implant: a multielectrode array implanted directly into the auditory nerve. We monitored the specificity of stimulation of the auditory pathway by recording extracellular unit activity at 32 sites along the tonotopic axis of the inferior colliculus. The results demonstrate the activation of specific auditory nerve populations throughout essentially the entire frequency range that is represented by characteristic frequencies in the inferior colliculus. Compared to conventional scala tympani stimulation, thresholds for neural excitation are as much as 50-fold
\end{abstract}

Correspondence to: John C. Middlebrooks • Kresge Hearing Research Institute, Department of Otorhinolaryngology - University of Michigan - Ann Arbor, MI 48109-0506, USA. Telephone: +1-7347637965; fax: +1-734-7640014; email: jmidd@umich.edu lower and interference between electrodes stimulated simultaneously is markedly reduced. The results suggest that if an intraneural stimulating array were incorporated into an auditory prosthesis system for humans, it could offer substantial improvement in hearing replacement compared to contemporary cochlear implants.

Keywords: auditory nerve, cat, cochlear implant, cochlear nerve, intraneural electrical stimulation, inferior colliculus

\section{INTRODUCTION}

A conventional cochlear implant consists of an array of platinum-iridium stimulating electrodes positioned along a space-filling carrier located within the scala tympani of the cochlea. The intended target of stimulation is either the auditory-nerve cell bodies in the spiral ganglion, lying within Rosenthal's canal, or the fibers of the auditory nerve, located within the central modiolus of the cochlea. The current path from scala tympani stimulating electrodes to excitable neural elements is subject to shunting by the surrounding volume of electrically conductive perilymph and to attenuation by the semiporous bone of the osseous spiral lamina. This spatial separation between electrodes and neurons precludes lowthreshold activation of frequency-specific neural populations. Moreover, scala tympani arrays typically are restricted to the basal turn and part of the middle turn of the cochlea, representing high and middle frequencies no lower than $\sim 600 \mathrm{~Hz}$ (Skinner et al. 2002; Wardrop et al. 2005). That is well above the 
$100-250-\mathrm{Hz}$ fundamental frequency of the human voice. The electrical current path from scala tympani arrays to low-frequency fibers of the cochlear apical turn is indirect, and electrical stimulation of those apical fibers is accompanied unavoidably by the activation of middle-to-high-frequency fibers.

An alternative to scala tympani electrode arrays for cochlear stimulation would be a multielectrode array penetrating directly into the auditory nerve. Intraneural stimulation was tested early in the history of auditory prosthesis (Simmons et al. 1964; Simmons et al. 1965; Simmons 1966; reviewed by Arts et al. 2003) but was abandoned in favor of scala tympani stimulation. We have revisited intraneural stimulation, in this case employing physiological procedures in anesthetized cats to characterize the selectivity of activation of the ascending auditory pathway. We stimulated the auditory nerve with a 16 -site penetrating electrode array oriented perpendicular to the modiolar trunk of the nerve. In each animal, we also tested responses to acoustic tones and to electrical stimulation with a conventional scala tympani electrode array. We monitored frequency-specific activation of the auditory pathway by recording from the central nucleus of the inferior colliculus (ICC), which contains a tonotopic representation of increasing frequency with increasing recording depth (Rose et al. 1963). The ICC recordings employed a multisite silicon-substrate recording probe that permitted simultaneous recording of single- and multiunit activity from 32 sites along the tonotopic axis. The distribution of activated neurons in the ICC reflects the frequency-specific information that is fed forward from the auditory nerve along the central auditory pathways.

The results demonstrate that intraneural electrodes can produce low-threshold stimulation of frequencyspecific neural populations, including specific stimulation of low-frequency fibers that are not directly accessible by scala tympani electrodes. Associated with restricted stimulation was a substantial reduction in interference between simultaneously stimulated pairs of electrodes. We discuss the implications of these findings for a new mode of auditory prosthesis for profoundly or severely deaf people.

\section{METHODS}

Experiments were conducted in barbiturate-anesthetized cats. Each experiment involved the following steps: (1) A multisite recording probe was implanted in the ICC. (2) ICC responses to acoustical tones in normalhearing conditions were recorded. Based on the responses to tones, the position of the recording probe was adjusted to sample a range of characteristic frequencies (CFs) from $<1$ to $>32 \mathrm{kHz}$, and then the probe was fixed in place. (3) The animal was deafened, and a conventional multichannel scala tympani stimulating electrode array was implanted. Responses of the ICC were recorded in response to single electrical pulses delivered through each of the electrodes of the scala tympani array. (4) The scala tympani array was removed and a multisite siliconsubstrate stimulating array was inserted into the modiolar trunk of the auditory nerve. Responses of the ICC were recorded in response to single electrical pulses delivered through each of the sites along the intraneural stimulating array. Successful acute experiments were conducted in 12 cats, 10 of which yielded responses to acoustic, scala tympani, and intraneural stimulation; two of the cats yielded only acoustic and scala tympani responses.

Stimulus presentation and data acquisition used System 3 equipment from Tucker-Davis Technologies (TDT; Alachua, FL, USA) and custom software running in MATLAB (The MathWorks, Natick, MA, USA). The electrical pulses were generated by custom optically isolated 8- or 16-channel current sources controlled by 16-bit digital-to-analog converters (TDT RX8). The 8-channel current source used for the scala-tympani stimulation had a maximum output of $3 \mathrm{~mA}$. The 16-channel current source used for the intraneural stimulation had a maximum output of $1 \mathrm{~mA}$.

Recording probes (NeuroNexus Technologies, Ann Arbor, MI, USA) consisted of single silicon-substrate shanks, $15 \mu \mathrm{m}$ thick and a maximum of $240 \mu \mathrm{m}$ wide, tapering toward the tip. Each probe had 32 iridium-plated recording sites, $413 \mu^{2}$ in area, arrayed in a line at $100-\mu \mathrm{m}$ intervals. The 32 -channel neural waveforms were digitized simultaneously, displayed online, and stored on computer disk. Offline, the waveforms were processed to remove interchannel correlated noise, and then single- or multiunit spikes were detected based on spike wave shapes (Middlebrooks 2004). Well-isolated single units, characterized by discrete clusters in histograms of peak-totrough spike amplitudes, were recorded at $11.6 \%$ of recording sites. All other recordings that were used for quantitative analysis consisted of spikes from unresolved clusters of two or more units. In response to stimulation with single electrical pulses at saturating levels, single units typically responded with mean spike rates of one to five spikes per trial; saturated multiunit responses typically averaged 5-10 spikes per trial. Spike counts were obtained in a time window of 2 to $15 \mathrm{~ms}$ relative to the onset of a single electrical pulse or 5 to $40 \mathrm{~ms}$ relative to the onset of a 40-ms tone burst. Those time windows were selected based on the inspection of poststimulus-time histograms in pilot experiments. 
The right ICC was visualized by aspiration of overlying occipital cortex. Stimuli were presented to the left ear; the right ear was deafened by the removal of the ossicular chain. One recording probe was inserted in each animal, oriented in the coronal plane and $40^{\circ}$ from the sagittal plane, passing dorsolateral to ventromedial through the ICC. That orientation corresponds approximately to the tonotopic axis of low-to-high CF in the ICC (Rose et al. 1963). Acoustical stimuli were presented through a calibrated hollow ear bar. Acoustic tones were $40 \mathrm{~ms}$ in duration with 5-ms rise/fall times; the levels of tone stimuli were calibrated from 0.5 to $40 \mathrm{kHz}$. Frequency response areas were compiled from responses to 5 to 10 repetitions of tones in $1 / 6$ octave intervals and sound levels in 5-dB intervals. The $\mathrm{CF}$ at each recording site was the frequency at which neurons responded with the lowest threshold. The recording probe was placed at the desired location relative to the frequency representation based on responses to tones, and then the probe was fixed in place. Upon completion of the presentation of acoustic stimuli, the left cochlea was deafened by scala tympani infusion of an ototoxic agent, neomycin sulfate $(10 \% \mathrm{w} / \mathrm{v})$ (Nuttall et al. 1977). In our pilot tests, this procedure eliminated all auditory responses measurable by compound-actionpotential or ICC-unit recording within $\sim 10 \mathrm{~min}$.

Scala tympani stimulating arrays were an animal version of the clinical Nucleus22 banded electrode array (Cochlear, Ltd., Lane Cove, NSW, Australia), differing from the human version only in that the array was truncated to eight electrodes. The electrodes each were $400-\mu \mathrm{m}$-diameter bands, arrayed on a silicone-elastomer carrier, centered at $750-\mu \mathrm{m}$ intervals. The electrode arrays were inserted through the round window as far as possible into the scala tympani; i.e., until the most apical electrode wedged into the scala. Because the scala tympani arrays were inserted until they would go no further, the apical electrodes probably achieved a tighter fit in the scala tympani, and closer proximity to the modiolus, than typically is achieved in human patients with banded electrode arrays. We estimate that the scala tympani arrays in the present experiments occupied all of the first and the basal half of the second turns of the scala tympani.

The intraneural stimulating arrays consisted of NeuroNexus probes similar to the recording probes. The stimulating arrays had 16 iridium-plated sites, $703-\mu \mathrm{m}^{2}$ in area, arrayed at $100-\mu \mathrm{m}$ intervals spanning a distance of $1.5 \mathrm{~mm}$ along a single, $15-\mu \mathrm{m}$-thick silicon-substrate shank. The intraneural array was implanted by enlarging the round window margin, then making a hole in the osseous spiral lamina with the bevel of a 30-gauge hypodermic needle and advancing the stimulating array with a microposi- tioner. The array was oriented approximately in the coronal plane, $\sim 45^{\circ}$ from the horizontal plane, passing from ventrolateral to dorsomedial. That orientation was approximately perpendicular to the midmodiolar axis of the cochlea. Several placements of the intraneural array near that general orientation were tested in each animal. In most of the successful array placements, all 16 stimulation sites lay within excitable neural tissue, indicating a $>1.5$ - $\mathrm{mm}$ traverse of excitable tissue. Summary data (e.g., spread of activation in Fig. 7) represent all the tested stimulating sites from a single array placement in each cat. The representative array placement selected from each cat was the one that gave the broadest range of activation of the ICC frequency representation; in some cases, all 16 stimulation sites were tested, whereas in other cases, only eight sites were tested, at $200-\mu \mathrm{m}$ intervals.

Scala tympani electrodes were stimulated in two configurations. In the monopolar configuration, the active electrode consisted of one scala tympani band and the return electrode was a wire in a neck muscle. In the bipolar configuration, the active electrode was one of the scala tympani bands and the return electrode was the adjacent more-apical scala tympani band. In most conditions, electrical stimuli for scala tympani and intraneural stimulation were single biphasic pulses, $41 \mu$ s per phase, initially cathodic. One exception was the situation in which thresholds for scala tympani stimuli were so high that it was necessary to lengthen the phase duration to $205 \mu$ s to achieve adequate stimulus levels. In that condition, the reported currents are multiplied by $205 / 41$ to permit an equivalent-charge comparison with stimuli that used $41-\mu$ s phase durations. That procedure was used for monopolar stimulation in one animal and bipolar stimulation in three animals; the example illustrated in Figure 2 used the standard phase duration of $41 \mu$ s per phase. The only other exceptions were the tests of two-pulse temporal integration presented in Figures 11 and 12 and accompanying text. In that condition, stimuli were pairs of monophasic cathodic pulses, $20 \mu$ s per phase. All intraneural stimulation employed a monopolar configuration, with single electrode sites on the multielectrode arrays serving as the active electrodes and a wire in a neck muscle serving as the return electrode.

The sensitivity of neural spike counts to changes in stimulus level was quantified by a procedure derived from signal detection theory (Green and Swets 1966; Macmillan and Creelman 2005). The stimulus-driven growth of response at each of the 32 ICC recording sites was represented by the discrimination index $\left(d^{\prime}\right)$ for the discrimination of stimulus levels differing by $5 \mathrm{~dB}$ (for sounds) or 1-2 dB (for electrical pulses); 
a no-stimulus condition was also included in each stimulus set. Based on responses on 20 trials of a given stimulus level and 20 trials of the next higher level, we formed an empirical receiver operating characteristic (ROC) curve based on the trial-by-trial distribution of spike counts among lower- and higher-level trials. The area under the ROC curve gave the probability of correct discrimination, which was expressed as a standard deviate (z-score) then multiplied by $\sqrt{ } 2$ to obtain $d^{\prime}$. This procedure was repeated for each successive pair of stimulus levels. The contours in the figures show $d^{\prime}$ cumulated across increasing stimulus levels. Threshold was taken as the interpolated stimulus level at which cumulative $d^{\prime}=1$. The threshold obtained from cumulating $d^{\prime}$ over successively increasing stimulus levels gave essentially the same value as that obtained from the comparison of various stimulus levels to a no-stimulus condition; across 2,468 cases of recording site and stimulating electrode, $98.5 \%$ showed a difference between the two procedures of no greater than $1 \mathrm{~dB}$ (mean $=0.079$
$\mathrm{dB})$. The advantage of using cumulative $d^{\prime}$ in this way is that it gave a measure of the ability of neurons to signal increasing stimulus levels well beyond the stimulus level at which stimuli could be detected almost perfectly (i.e., beyond $d^{\prime}=3.3$ ). This signaldetection procedure was favored over, for instance, a measure of spike rate divided by standard deviation of the rate because the signal-detection procedure explicitly incorporated the trial-by-trial variation in spike rates for both lower- and higher-level stimuli. Also, values expressed as $d^{\prime}$ lend themselves to comparison with psychophysical results.

\section{RESULTS}

Each animal was tested first in normal-hearing conditions with acoustic tone stimulation. Then, the animal was deafened and tested with single-pulse electrical stimulation, first with a conventional scala
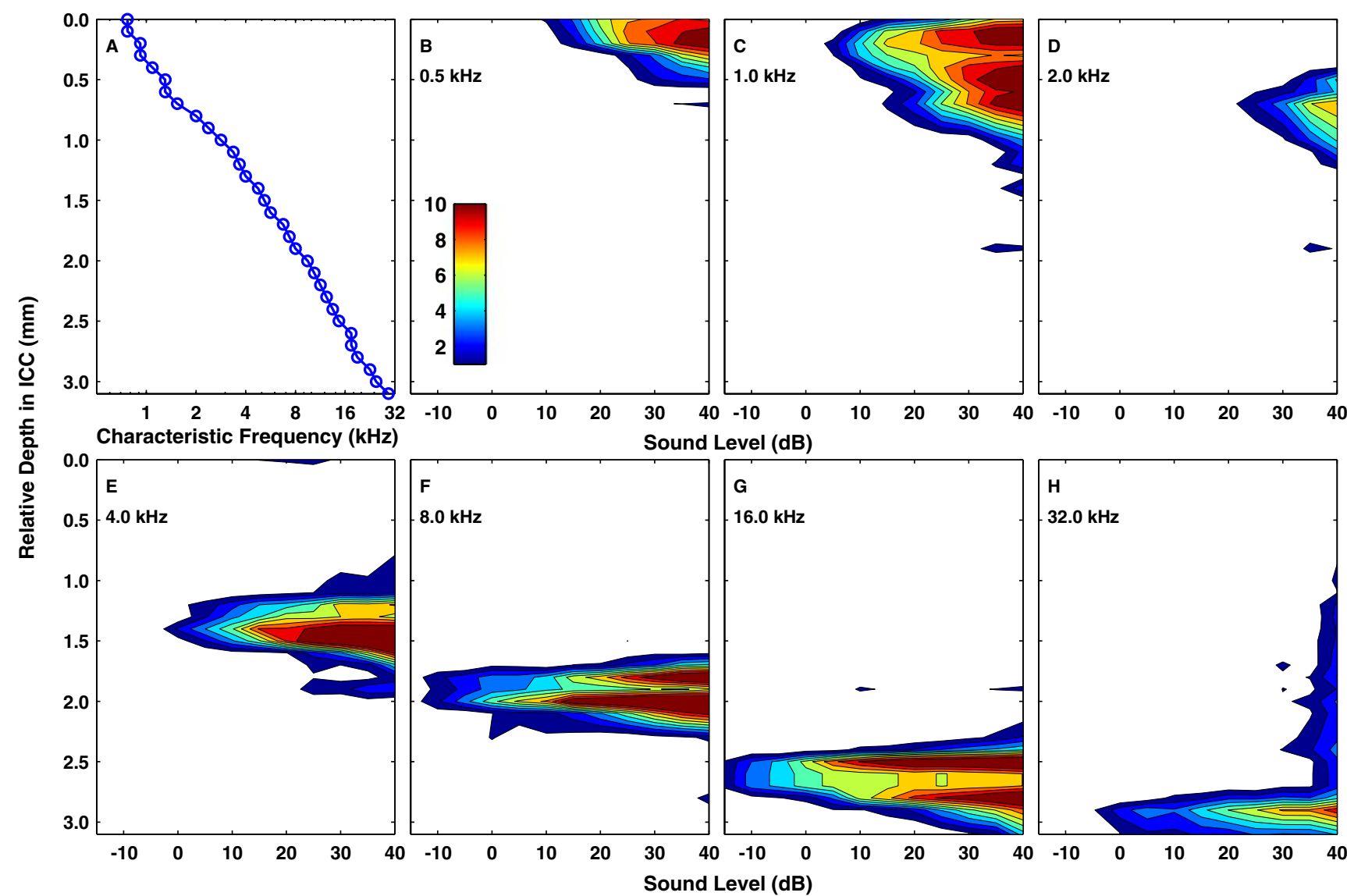

FIG. 1. Responses to acoustic tones. (A) CFs for single- and multiunit activity were recorded simultaneously at 32 depths in the ICC. The independent variable, ICC depth, is plotted on the vertical axis to permit comparison with panels (B) through (H) and subsequent figures. (B)- $(\mathrm{H})$ "Spatial tuning curves" showing the distribution of activity in the ICC elicited by tones as a function of sound level. Each panel represents the response to tone of a single frequency (as indicated). Contours represent cumulative discrimination index $\left(d^{\prime}\right)$ in steps of one $d^{\prime}$ unit. The entire colored area in each panel represents the range of ICC depths at which the tone produced activity distinguishable from background with a criterion of $d^{\prime} \geq 1$. Cat0509. 


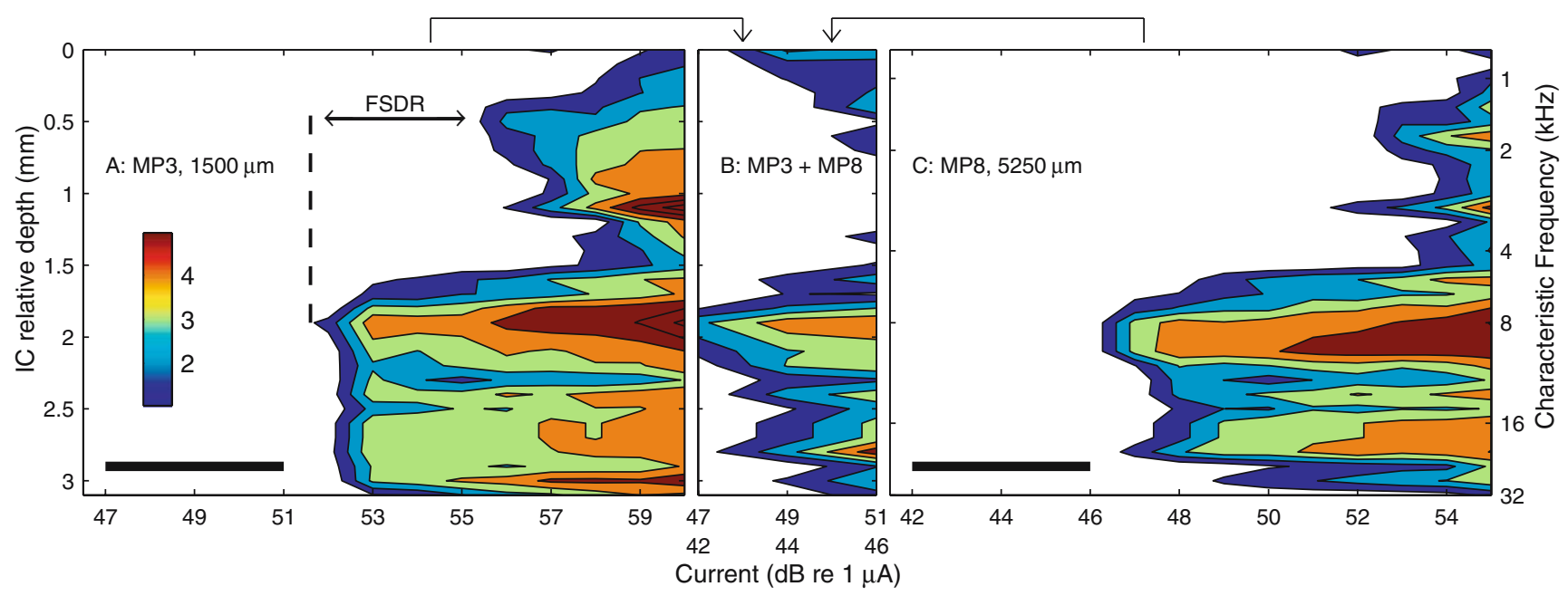

FIG. 2. Spatial tuning curves elicited by individual and simultaneous monopolar stimulation of two scala tympani electrodes. The left ordinate axis plots recording depth in the ICC relative to the most superficial recording site; corresponding CFs are plotted on the right ordinate. The abscissa plots the current level of single electrical pulses. (A) and (C) Responses to monopolar stimulation of scala tympani electrodes 3 and 8 , respectively, stimulated individually in monopolar configuration. Electrode 8 was centered in the scala tympani $3.75 \mathrm{~mm}$ more apical than electrode 3. (B) Activity elicited by simultaneous stimulation of the two electrodes. Stimulus currents on the two electrodes were varied in parallel; upper and lower rows of abscissa labels refer to currents presented to the electrodes represented in panels (A) and (C), respectively. The current ranges used in the simultaneous condition (indicated with black bars in panels (A) and (C)) were entirely below the thresholds for activation with either electrode alone. FSDR is defined in the text. Cat0509.

tympani banded electrode array and then with the intraneural array. Figures 1, 2, 5, and 8 are based on recordings from the same animal and same ICC recordings sites so that responses can be compared across the various stimulation modalities. Observations in that animal are compared with other individual examples (Figs. 6, 9, and 11) and with population data (Figs. 7, 10, and 12). We begin by describing baseline results to acoustical and conventional scala tympani stimulation. Next, we describe the basic characteristics of responses to intraneural stimulation. Finally, we compare scala tympani and intraneural stimulation in regard to the problem of interference among electrodes.

\section{Responses to acoustical and conventional scala tympani electrical stimulation}

Responses in the ICC to acoustic tones in normalhearing conditions showed the well known CF-vs-ICCdepth (tonotopic) organization (Fig. 1A). The recording probe was positioned to sample ICC neurons ranging in $\mathrm{CF}$ from $<1$ to $\sim 32 \mathrm{kHz}$. Single tones activated spatially restricted populations of neurons distributed along the tonotopic axis of the ICC. The distribution of ICG activity along the tonotopic axis as a function of stimulus level is represented by "spatial tuning curves" (Fig. 1B-H); the tuning curves are "spatial" in the sense of space in the ICC. Each of these plots represents the sensitivity of ICC neurons at a single tone frequency. Contours represent the cumulative discrimination index (cumulative $d^{\prime}$ ) for the discrimination of successive 5-dB increases in sound level based on level-dependent changes in trial-by-trial spike count at single recording sites. The contours are drawn in steps of one $d^{\prime}$ unit. Some of the spatial tuning curves (e.g., Fig. 1F, G) show a bimodal pattern, which we presume is due to differences among neurons in their growth of activity with increasing stimulus strength. The overall vertical extent of the colored area at each sound level represents the distribution in the ICC of suprathreshold neural activity at each sound level; a criterion of $d^{\prime}=1$ is taken as threshold. The population of neurons activated by a single tone showed modest expansion with increasing level of a tonal stimulus. Spatial tuning curves shifted systematically to deeper ICC locations as tone frequencies were increased. The responses elicited by tones provided a tonotopic reference and a basis for comparison with later measures of electrically stimulated activity.

After mapping with tones in each animal, the recording probe was fixed in place and the left cochlea (contralateral to the ICC recording sites) was deafened by infusing neomycin sulfate into the scala tympani. A conventional eight-banded-electrode array (i.e., a scala tympani cochlear implant) was inserted in the scala tympani through the round window. Single electrical pulses were presented 
through the scala tympani electrodes, and ICC responses were recorded. A monopolar electrode configuration was employed in which each scala tympani electrode could serve as the active stimulating electrode, and a ground wire inserted in a neck muscle provided the electrical return. Monopolar is the configuration used in the majority of contemporary cochlear prosthesis systems. The illustrated example (Fig. 2) shows responses elicited by two scala tympani electrodes. Monopolar scala tympani stimulation resulted in much broader spread of excitation than that produced by any acoustical tone (Fig. 2A, C; Fig. 2B is discussed in a later section). In these examples, stimulation at the lowest levels led to the activation of ICC neurons previously shown to have CFs ranging from $\sim 6$ to $29 \mathrm{kHz}$, and a 3-6-dB increase in current led to the nonspecific activation of ICC neurons across the entire frequency representation. We presume that the activation of the low-frequency neurons in the dorsal ICC is due to the current from scala-tympani electrodes in the cochlear base spreading to activate fibers from the cochlear apex as they travel past the basal turn in the modiolus.

We define the frequency-specific dynamic range (FSDR) as the range of currents within which there was no spread of activation to noncontiguous frequency ranges. The spatial tuning curve Figure 2A shows a discrete low-frequency lobe centered at ICC depth $0.5 \mathrm{~mm}$. The FSDR in that case was $3.7 \mathrm{~dB}$ measured from the threshold at the main lobe $(51.7 \mathrm{~dB}$ at depth $1.9 \mathrm{~mm})$ to the threshold of the noncontiguous lobe $(55.4 \mathrm{~dB}$ at depth $0.5 \mathrm{~mm})$. The FSDR was $5.2 \mathrm{~dB}$ in the case shown in Figure 2C. In some other cases, thresholds fell off continuously from the most sensitive ICC depth to the two ends of the recording array (i.e., depths 0 and $3.1 \mathrm{~mm}$ ). In those cases, we measured the FSDR at the lower of the thresholds at the two ends. In each of the 12 animals that were tested with monopolar scala tympani stimulation, we identified the stimulating electrode that gave the greatest FSDR. Across the 12 animals, the median value of the maximum FSDR was $3 \mathrm{~dB}$; the range in 11 animals was 0 to $7 \mathrm{~dB}$, with one animal showing no low-frequency activation at any tested level. We also tested a bipolar configuration in which the active electrode was one scala tympani electrode and the return was the adjacent scala tympani electrode. In 11 animals tested with bipolar stimulation, four animals showed maximum FSDRs ranging from 0 to $10 \mathrm{~dB}$, and the other seven animals showed activation restricted to high-frequency regions with no response in low-frequency regions at any tested level. In this and in previous studies of scala tympani stimulation using animal models (Bierer and Middlebrooks 2002; Snyder et al. 2004), we have been unable to activate the low-frequency representation in the ICC or auditory cortex without also activating the high-frequency representation at equal or lower current levels. That is, any activation of apical fibers that we have observed in response to scala tympani stimulation has been nonspecific, reflecting a breakdown in the specificity of stimulation of high-frequency (basal) fibers.

\section{Responses to intraneural stimulation}

The intraneural stimulating electrode array was positioned in the modiolar portion of the auditory nerve using a lateral approach through the round window. Figure 3A shows the relevant structures in a postmortem dissection. The membrane of the round window has been removed, affording a view into the hook and beginning of the basal turn regions of the scala tympani. The black arrow indicates the location of the habenula at the edge of the osseous spiral lamina. The white arrow indicates a gray curve corresponding to the location of Rosenthal's canal, site of the spiral ganglion. The white circle below the line of Rosenthal's canal indicates the approximate location of openings that we made through the osseous spiral lamina for the passage of intraneural stimulating arrays into the modiolar trunk of the auditory nerve. Figure $3 \mathrm{~B}$ shows an in vivo view of a silicon-substrate stimulating array inserted through the osseous spiral lamina. The round-window margin has been enlarged ventrally and laterally-its original perimeter is indicated by the dashed line. The stimulating array is foreshortened in this view and the stimulating sites are hidden within the nerve. The inset shows a stimulating array with its 16 sites visible.

A schematic of the auditory nerve and cochlear nucleus is shown in Figure 4. It shows the spiral course of auditory nerve fibers exiting Rosenthal's canal and entering the modiolar trunk of the nerve. That spiral organization results in fibers from apical, middle, and basal turns lying, respectively, at deep, intermediate, and superficial positions in the nerve trunk, as indicated by cross-sections I and II. Our intraneural stimulating arrays were oriented perpendicular to the long axis of the nerve approximately at the level of cross-section II in the illustration. Based on the progression of cochlear-place-specific responses to electrical stimulation, we infer that most of our stimulation sites lay along the line segment drawn in cross-section II.

Stimulation with electrodes implanted within the auditory nerve provided more specific activation of neural populations than was possible with the scala tympani array. Figure 5 shows spatial tuning curves recorded in the ICC in response to individual stimulation at eight intraneural electrode sites, spaced 

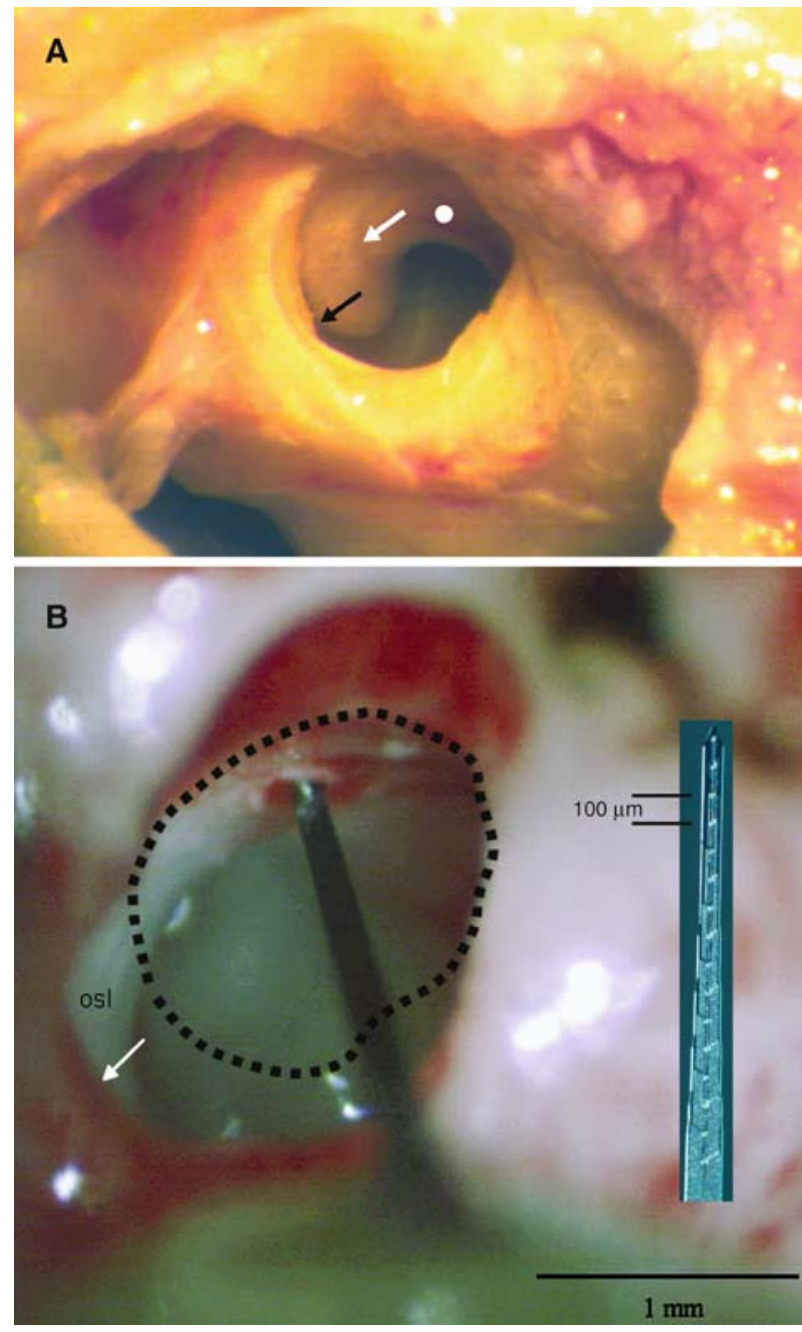

FIG. 3. Access to the auditory nerve. (A) Lateral view of the region of the round window in a postmortem dissection. The black arrow indicates the margin of the osseous spiral lamina, the location of the basilar membrane. The white arrow indicates a curving gray shadow corresponding to the location of Rosenthal's canal. The white circle indicates the approximate location, below Rosenthal's canal, at which small holes were made in the osseous spiral lamina to permit access to the modiolar trunk of the auditory nerve. (B) In vivo view of a silicon-substrate stimulating probe inserted into the auditory nerve through a hole in the osseous spiral lamina. The dashed line indicates the approximate location of the round-window perimeter prior to surgical enlargement. The inset shows a photograph of a stimulating array at the same scale as the in vivo image.

at 200- $\mu \mathrm{m}$ intervals through the modiolar trunk of the auditory nerve; stimulation sites are labeled according to depth in the auditory nerve relative to the most proximal (i.e., superficial) electrode site. The ordinate on the right indicates the CFs that were obtained using acoustic tones. Activation of each intraneural electrode produced a distinct pattern of activity in the ICC. One can see examples of specific activation of low-, middle-, and high-frequency ICC regions (Fig. 5D, A, and H, respectively), spanning essentially the entire range of frequencies that is represented by CFs recorded in the ICC. Other stimulation sites activated two discrete frequency regions with equal thresholds (Fig. 5F), or produced rather broad low-to-mid-frequency activation (Fig. 5B). Examples of spatial tuning curves elicited by intraneural stimulation in another animal are shown in Figure 6; the stimulating sites in this figure are reordered according to the procedure described in a following paragraph.

The spread of excitation produced by intraneural stimulation generally was more restricted than that produced by scala tympani stimulation and, in many instances, was comparable in extent to the spread of excitation produced by pure tones in normal-hearing conditions. We inferred the spread of auditory nerve excitation by the spread of activation along the tonotopic axis of the ICC. The spread of activation was quantified by the percentage of ICC recording sites that were active (according to a criterion of cumulative $\left.d^{\prime} \geq 1\right)$ at various specified stimulus levels. Given a recording array comprising 32 sites spaced at $100-\mu \mathrm{m}$ intervals, activation of $25 \%$ of the sites,

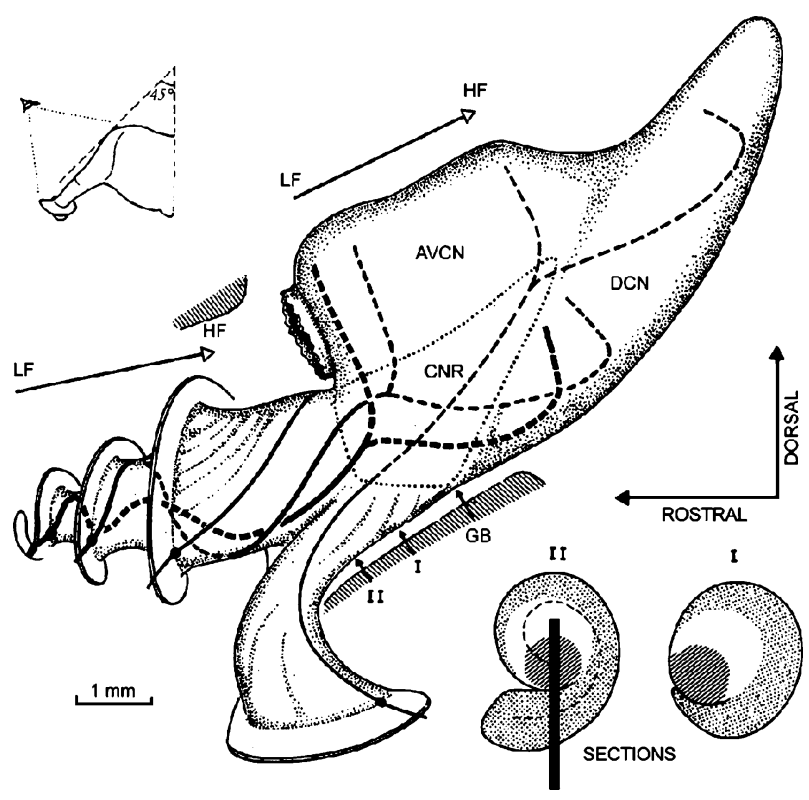

FIG. 4. Topography of cochlear nerve and nucleus in the cat (reprinted from Arnesen and Osen, 1978 with permission from the publisher). The projections from the apical, middle, and basal turns to the cochlear nucleus are indicated with lines varying in thickness. The two cross sections (lower right, labeled I and II) show the approximate locations within the nerve of fibers from various cochlear turns. Medium, clear, and dense shading indicate fibers from the basal, middle, and apical turns, respectively. The line in cross section II indicates a typical placement of the stimulating array, estimated on the basis of frequency-specific responses recorded in the ICC and post-mortem dissections. Abbreviations: AVCN: anterior ventral cochlear nucleus; DCN: dorsal cochlear nucleus; CNR: cochlear nerve root; GB: glial border; LF: low frequency; HF: high frequency. 

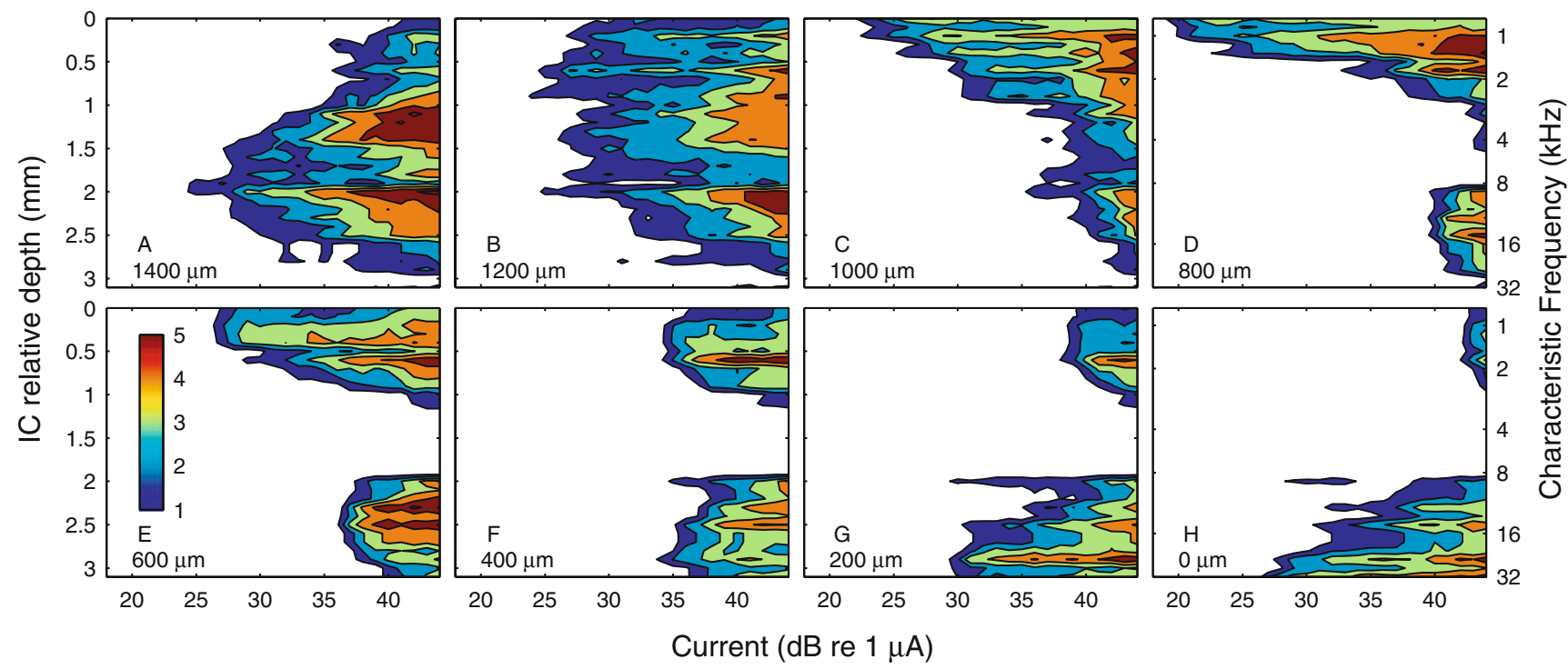

FIG. 5. Spatial tuning curves elicited by intraneural stimulation. Each panel corresponds to one stimulus site, labeled according to depth relative to the most proximal (i.e., superficial) stimulation site. Illustrated examples are from 8 of the 16 available sites on the stimulating array. These spatial tuning curves appear somewhat patchier than those for acoustic stimulation in Figure 1, largely because these data for intraneural stimulation were collected at 32 stimulus levels in 1-dB steps, whereas the data for acoustic stimulation were collected at only 13 levels in $5-\mathrm{dB}$ steps, necessitating somewhat greater interpolation in the contour plots. Cat0509.

for instance, indicates a spread of activation across $800 \mu \mathrm{m}$ of the ICC tonotopic axis that we sampled. Stimulus levels in Figure 7A-C were specified relative to the threshold for the most sensitive ICC recording site (i.e., relative to the tip of the spatial tuning curve). Our measurements of spread of ICC activation in many cases underestimate the spread of auditory nerve excitation because spatial tuning curves often extended beyond the range of ICC sites sampled by the recording array. Nevertheless, that underestimate applied to all stimulus conditions, so it had relatively little effect on comparisons among conditions. The distribution of spread of activation among stimulation sites and animals is shown in the form of box plots in Figure 7. Figure 7A, B, and $\mathrm{C}$ show the distributions measured at current levels 3, 6, and $10 \mathrm{~dB}$ above threshold, respectively; Figure 7D is discussed in a following paragraph. There is a clear increase in the spread of activation between acoustic tones and intraneural electrical stimulation, between intraneural and bipolar scala tympani stimulation, and between bipolar and monopolar scala tympani stimulation. Each of those pair-wise comparisons showed a significant difference at each of the 3-, 6-, and 10-dB levels $(p<0.0001$; analysis of variance).

Direct comparisons of spread of activation among these various stimulus modalities must be interpreted with some caution because of the wide differences in dynamic ranges, especially the difference in dynamic range between acoustic stimulation and any of the electrical stimulus configurations. For instance, it might be more appropriate to compare the distribution of spread of activation for the intraneural condition at $3 \mathrm{~dB}$ above threshold with that for the
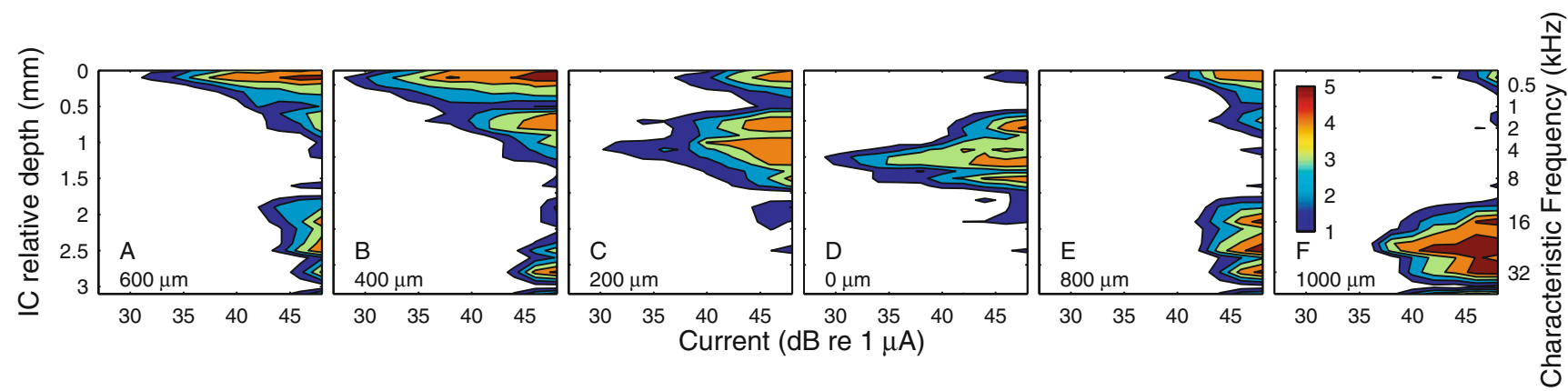

FIG. 6. Spatial tuning curves elicited by intraneural stimulation, sorted according to locus of ICC activation. Note that these data are from a different cat than that represented in Figure 5. Cat0502. 


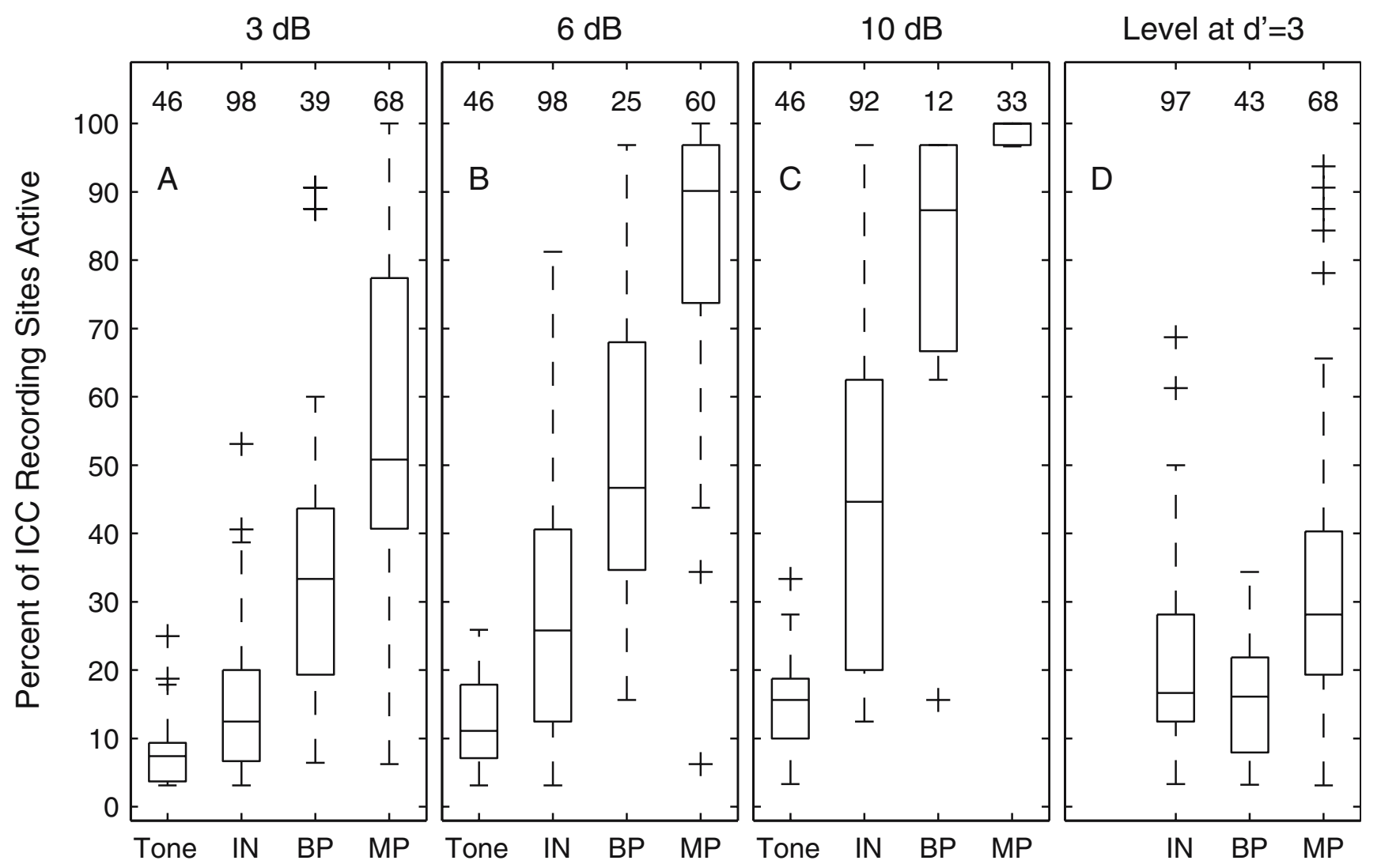

FIG. 7. Distributions of spread of ICC activation. These box-and-whisker plots show the distribution among animals and stimulating electrodes (or tone frequencies) of spread of ICC activation. The datum for each case is the percent of ICC recording sites at which units were active according to a criterion of $d^{\prime}=1$ in response to a stimulus at a particular level. The distribution of spread of activation for each stimulus condition is represented by one box and whiskers. The boxes have horizontal lines at the lower quartile, median, and upper quartile values. The whiskers show the extent of data lying within 1.5 times the interquartile distance from the ends of the boxes. Outlines are indicated by plus signs. The abscissa labels indicate the stimulus conditions: Tone for acoustic tones in one-octave steps from 0.5 or $1 \mathrm{kHz}$ to $32 \mathrm{kHz}$, IN for 8 to 16 intraneural stimulating sites, $B P$ for up to seven bipolar scala tympani electrodes, and $M P$ for up to eight monopolar scala tympani electrodes. (A), (B), and (C) show distributions for stimulus levels 3, 6, and $10 \mathrm{~dB}$, respectively, above the threshold for the most sensitive ICC recording site. (D) shows distributions for stimulus levels set to the level at which the cumulative $d^{\prime}=3$ for the most sensitive ICC site (see text). The number shown over each box indicates the number of cases in the corresponding distribution. Data for the IN and MP conditions are drawn from nine cats in which reliable spike activity was recorded at 27 to 32 recording sites; data from a 10th cat were excluded from this figure because only 12 recording sites were functional in that cat. Data for the $B P$ condition are drawn from the eight cats that were tested with bipolar stimulation. Data for the Tone condition in panels (A), (B), and (C) are drawn from six cats (in three of the nine cats, not enough stimulus repetitions were tested to permit detailed threshold determinations). Tone data are omitted from panel (D) because none of the cats were tested with enough repetitions to permit that analysis. The number of $B P$ data is small, particularly at the highest stimulus level, because thresholds for bipolar stimulation often exceeded the 3-mA limit of our stimulator.

tone condition at $10 \mathrm{~dB}$ above threshold. The distributions of activations by tones measured at 6 and $10 \mathrm{~dB}$ above threshold overlap extensively with the distribution of activations by intraneural stimulation measured at $3 \mathrm{~dB}$ above threshold.

Comparison of the intraneural spatial tuning curves in Figures 5 and 6 with the monopolar scala tympani tuning curves in Figure 2 suggests that dynamic ranges (i.e., the range of increasing stimulus levels over which neural responses increase) are greater for intraneural than for scala tympani stimulation. We seldom could be sure that we had reached saturating levels of stimulation because we often reached the 3-mA upper limit of the stimulator (most often in the case of bipolar stimulation) or we elicited unintended muscle activation (most often in the case of monopolar stimulation). For that reason, it was impractical to quantify complete dynamic ranges at all stimulus configurations and all ICC recording sites. As an alternative, we measured the difference in stimulus levels between the cumulative $d^{\prime}=1$ and $d^{\prime}=3$ contours measured at the ICC depth showing the lowest threshold. We then divided two $d^{\prime}$ units by the measured difference in stimulus levels to yield the "discrimination slope," expressed in units of $d^{\prime}$ units per decibel. The discrimination slope for intraneural stimulation was $0.73 d^{\prime}$ units per decibel (total of 96 stimulus sites in 
nine animals) compared with 1.98 and 1.94 for monopolar and bipolar scala tympani stimulation, respectively (66 stimulus sites in nine animals for monopolar, 38 sites in eight animals for bipolar). These values confirm a greater dynamic range for intraneural stimulation, at least for the initial slope of the stimulus-response function.

Figure 7D shows the distributions of a measure of spread of activation that attempts to adjust for the difference in discrimination slope among electrical stimulation configurations. For each spatial tuning curve, we identified the most sensitive ICC recording site, found for that site the stimulus level that produced discrimination at a cumulative- $d^{\prime}$ criterion of 3 , and identified the percentage of ICC recording sites that were active at that stimulus level (using a criterion of $d^{\prime} \geq 1$ ). That procedure resulted in the spread of activation measurements closer to the tips of spatial tuning curves for the two scala tympani conditions than for the intraneural condition. Measured in this way, the distributions of spread of activation for intraneural and bipolar stimulation overlapped broadly, with a weak but significant tendency for more restricted spread in the bipolar condition $(p<0.05$, analysis of variance). There still was a prominent difference between monopolar scala tympani and either intraneural or bipolar scala tympani stimulation $(p<0.0001$; analysis of variance).

In each animal, we attempted to identify examples of intraneural stimulation sites that yielded selective stimulation patterns in low-, middle-, and high-frequency regions of the ICC tonotopic axis; data from those stimulating electrodes are shown in Table 1. The frequency ranges chosen $(0.6-1.3,2-10$, and 23-
$38 \mathrm{kHz}$ ) correspond approximately to CFs of afferents from the apical, middle, and basal turns of the cochlea. Whenever possible, we selected stimulation sites for which spatial tuning curves peaked within the portion of the tonotopic axis sampled by our 32channel recording probe; recording sites typically covered a range of CFs from 0.5 to $1 \mathrm{kHz}$ at the lowfrequency end to $32-40 \mathrm{kHz}$ at the high-frequency end. Several stimulation sites were not selected because their spatial tuning curves appeared to peak at ICC tonotopic sites below the sampled frequency range (in 5 of 10 cats) or above the sampled range (in 3 of 10 cats). For that reason, the range of CFs represented in Table 1 understates the range of the frequency representation covered by intraneural stimulation sites. Frequency-specific stimulation sites in all three frequency ranges, low, middle, and high, could be identified in 6 of 10 animals-in two animals, we accessed selectively only middle- and high-frequency stimulation sites, and in two other animals we accessed only low- and middle-frequency sites. For the selected stimulation sites, we identified the $\mathrm{CF}$ at the center of the activated ICC locus (previously measured using tonal stimulation), the threshold for electrical stimulation, and the FSDR.

Intraneural stimulation consistently produced greater dynamic ranges for frequency-specific activation than did scala tympani stimulation, as indicated by median FSDRs of $16.5 \mathrm{~dB},>15.5 \mathrm{~dB}$, and $>12.5 \mathrm{~dB}$ for low-, middle-, and high-frequency intraneural stimulation sites. Those values contrast with the median value of $3 \mathrm{~dB}$ for obtained with monopolar scala tympani stimulation. We presume that the greater FSDRs for intraneural stimulation compared

TABLE 1

\begin{tabular}{|c|c|c|c|c|c|c|c|c|c|}
\hline \multicolumn{10}{|c|}{ Characteristics of selected low-, middle-, and high-CF stimulation sites } \\
\hline \multirow[b]{2}{*}{ Animal \# } & \multicolumn{3}{|c|}{ Low CF } & \multicolumn{3}{|c|}{ Middle CF } & \multicolumn{3}{|c|}{ High $C F$} \\
\hline & $C F$ & Thr & FSDR & $C F$ & Thr & FSDR & $C F$ & Thr & $F S D R$ \\
\hline 0501 & - & - & - & 6 & 28 & $>12$ & 32 & 33 & $>8$ \\
\hline 0502 & 0.7 & 31 & 14 & 5 & 29 & 15 & 23 & 32 & 15 \\
\hline 0503 & 0.8 & 20 & $>20$ & 3 & 25 & $>17$ & 32 & 26 & 12 \\
\hline 0505 & 0.8 & 23 & 9 & 2 & 30 & 15 & 27 & 25 & 19 \\
\hline 0507 & 1.0 & 42 & $>20$ & 10 & 42 & 19 & 32 & 39 & $>23$ \\
\hline 0509 & 0.8 & 22 & 18 & 10 & 24 & $>18$ & 29 & 26 & 15 \\
\hline 0510 & 0.7 & 35 & 15 & - & - & - & 38 & 33 & 12 \\
\hline 0512 & - & - & - & 4 & 26 & 18 & 29 & 28 & 13 \\
\hline 0513 & 0.6 & 28 & 7 & 6 & 30 & 7 & 25 & 33 & 5 \\
\hline 0601 & 1.3 & 20 & $>18$ & - & - & - & 32 & 30 & $>11$ \\
\hline Minimum: & 0.6 & 20 & 7 & 2 & 24 & 7 & 23 & 25 & 5 \\
\hline Median: & 0.8 & 26 & 17 & 6 & 29 & $>16$ & 31 & 31 & $>13$ \\
\hline Maximum: & 1.3 & 42 & $>20$ & 10 & 42 & 19 & 38 & 39 & $>23$ \\
\hline
\end{tabular}

$\mathrm{CF}$ (in $\mathrm{kHz}$ ) associated with the ICC locus activated at lowest current level. FSDRs shown with a greater-than (>) sign indicate cases in which there was no activation of noncontiguous frequencies at the highest current level that was tested.

Thr $=$ Current threshold $(\mathrm{dB}$ re. $1 \mu \mathrm{A})$. 
to scala tympani stimulation reflects both the lower rate of increase in activity with increasing stimulus level (given by the shallower discrimination slope) and the more restricted spread of excitation.

All of our results from intraneural stimulation showed a nonmonotonic relationship between depth in the auditory nerve and depth along the tonotopic axis of the ICC. This is expected from the spiral organization of fibers in the auditory nerve (Arnesen and Osen 1978). In the example in Figure 5, a deepto-superficial succession of stimulation sites activated a middle-frequency region $(\sim 9 \mathrm{kHz})$, then progressively lower frequency regions (to $<1 \mathrm{kHz}$ ), then a bilobed pattern of low-and-high-frequency regions, and finally a specific high-frequency region $(>32 \mathrm{kHz})$. Based on the pattern of ICC activation, we infer that the intraneural electrode array represented in Figure 5 passed first through high-frequency basalturn fibers located ventrally and superficially, then through low-frequency apical-turn fibers in the center of the nerve, and terminated in mid-frequency middle-turn fibers located just beyond the center of the nerve trunk. Intraneural electrode arrays in six animals accessed selective stimulation sites on all three of those frequency ranges, and each of those cases showed the same sequence of frequency regions: deep, intermediate, and superficial nerve stimulation sites activated ICG regions with, respectively, middle, low, and high CFs. Array placements in the other four animals all accessed high-frequency fibers from the cochlear base, which lie superficial in the nerve, but either terminated amid the lowfrequency apical fibers in the center of the nerve or bypassed the apical fibers and accessed only middlefrequency fibers.

If intraneural stimulation were to be adapted for use in a prosthetic device in humans, a functional procedure would be necessary for "unwrapping" the nonmonotonic relationship between depth in the nerve and frequency. We implemented a simple automated procedure in which stimulating electrodes were reordered based on the centroid of activity along the tonotopic axis of the ICC. The results of that procedure are shown in Figure 6. The spatial tuning curves show a monotonic progression from low- to high-CF regions of the ICC, but the depths of nerve stimulation sites indicated in each panel indicate that the sites have been reordered. One of the stimulation sites, at $800 \mu \mathrm{m}$ (Fig. 6E), elicited a bilobed ICC response pattern. The position assigned to that site by the reordering algorithm was rather sensitive to parameters of the algorithm; similar behavior was noted for bilobed sites when reordering the case shown in Figure 5. In a practical prosthesis application, we speculate that stimulation sites like that at depth $800 \mu \mathrm{m}$ in Figure $6 \mathrm{E}$ might lead to ambiguous percepts that one might opt to eliminate from a sound-processing program; the elimination of unsatisfactory channels is a standard practice with conventional cochlear implants.

Thresholds obtained with intraneural stimulation were significantly lower than those obtained using a conventional scala tympani electrode array. We compared scala tympani stimulation using monopolar and bipolar electrode configurations and (monopolar) intraneural stimulation. In each cat, we estimated the threshold for the most sensitive stimulating electrode in each condition. Across the nine cats that were tested with all three stimulus conditions, mean thresholds were 60.3, 50.7, and $26.2 \mathrm{~dB}$ (re: $1 \mu \mathrm{A})$ for bipolar scala tympani, monopolar scala tympani, and intraneural stimulation, respectively $(p<0.001$ for all intercondition comparisons, two-tailed paired $t$ test).

\section{Responses to simultaneous two-channel stimulation}

One challenge of multichannel auditory prosthesis design is to transmit information across a large number of functionally independent channels. In our animal model, the stimulation of individual scala tympani electrodes led to the activation of largely overlapping ICC neural populations, as in Figure 2A, C. We also tested a condition in which pulses were presented simultaneously through two active electrodes. In the case illustrated in Figure 2B, stimulus levels on each electrode (indicated by black bars in Fig. 2A, C) were below the thresholds for either electrode alone. Simultaneous stimulation resulted in wide-spread ICC activation at a threshold $>4 \mathrm{~dB}$ lower than the thresholds for single-electrode stimulation. That result indicates that these two electrodes were functionally dependent in that low-level stimulation of one electrode resulted in either no response or widespread activation, depending on the absence or presence of low-level stimulation of the other electrode.

The simultaneous activation of various pairs of scala tympani electrodes was studied in four animals. For each pair of stimulating electrodes, we stimulated each electrode individually and then stimulated the two electrodes simultaneously with identical currents. We identified the electrode exhibiting the lower threshold in the single-electrode condition and compared that threshold with the threshold obtained in the simultaneous-stimulation condition; in each case, thresholds were measured at the most sensitive ICC depth. In each case of scala tympani stimulation, there was substantial overlap between the two individual spatial tuning curves. Across a total of 16 pairs of stimulating electrodes (one, one, seven, or seven pairs in each of four cats), thresholds were reduced 


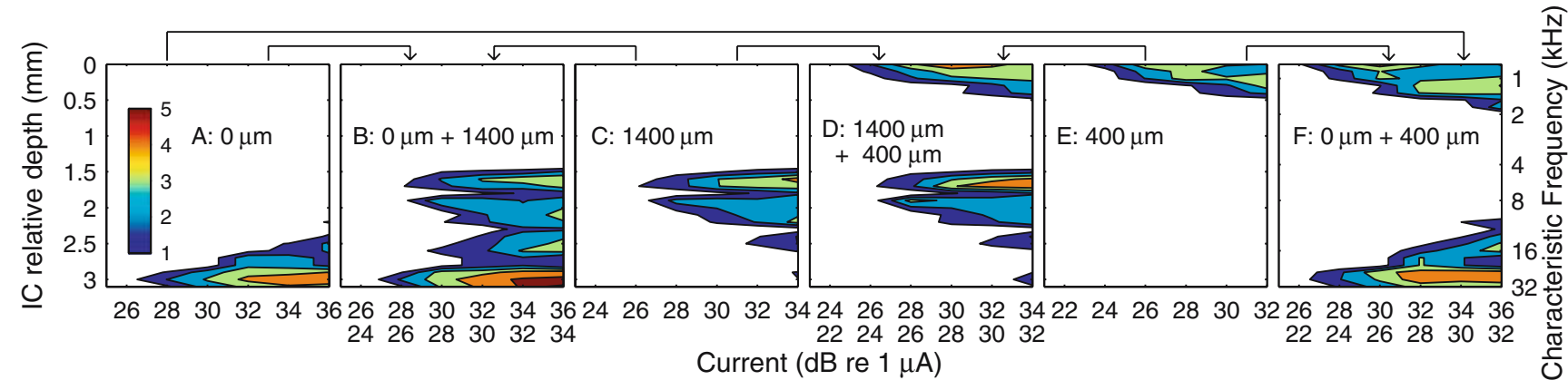

FIG. 8. Simultaneous stimulation of intraneural electrodes. (A), (C), and (E) show spatial tuning curves elicited by individual stimulation of three intraneural electrode sites. Stimulation sites have been ordered to show a high-to-low-frequency progression from left to right. (B), (D), and (F) show spatial tuning curves elicited by single pulses presented simultaneously to various pairs of electrode sites. As in Figure 2, the currents to the two electrodes were varied in parallel, although in this case, the current levels were the same as the suprathreshold levels used in panels (A), (C), and (E). Cat0509.

by $4.2 \pm 1.2 \mathrm{~dB}$ in the two-electrode condition compared to the more sensitive of the single-electrode conditions.

Intraneural stimulation showed considerably less interference among simultaneously stimulated electrodes. In one illustrated example, the individual stimulation of three intraneural electrodes resulted in three largely nonoverlapping activated ICC neural populations (Fig. 8A, C, E). The simultaneous stimulation of pairs of those intraneural electrodes at the same levels used for the single-electrode conditions produced superimposed ICC activation patterns (Fig. 8B, D, F). In the paired conditions, the contribution of each individual electrode is clear, and there are negligible shifts in the thresholds for the activation of each electrode. That is, the response to each intraneural electrode was essentially independent of stimulation of other intraneural electrodes. An example from another animal is shown in Figure 9A-C. The stimulating electrodes in this case were only $200 \mu \mathrm{m}$ apart. Again, there was little overlap between the excitation patterns elicited by the two electrodes, and the stimulation of each electrode had little influence on the pattern elicited by the other electrode.

A greater degree of interaction between intraneural electrodes was observed in instances in which there was greater overlap of excitation patterns. In the example in Figure 9D-F, stimulating electrodes were $600 \mu \mathrm{m}$ apart, but they activated largely overlapping neural populations in the ICC (and presumably overlapping populations in the auditory nerve). Simultaneous stimulation of both electrodes resulted in a threshold $\sim 4 \mathrm{~dB}$ lower than the threshold for either electrode alone.

We quantified threshold effects of simultaneous intraneural stimulation in five cats $(1,3,4,9$, or 21 electrode pairs per cat; total of 38 pairs). For 23 pairs of stimulating electrodes which evoked largely non- overlapping spatial tuning curves, as in Figures 8 and 9A-C, threshold reductions due to simultaneous stimulation were small, averaging $0.3 \pm 0.9 \mathrm{~dB}$ (mean \pm $\mathrm{SD})$. In 15 cases in which there was appreciable overlap of spatial tuning curves, as in Figure 9D-F, threshold reductions were appreciably greater, averaging $3.6 \pm 1.2 \mathrm{~dB}$.

We explored the mechanisms underlying simultaneous-stimulation threshold reductions by quantifying threshold reductions at individual ICC recording sites. For each ICC recording site and each pair of stimulating electrodes, we computed the difference in thresholds obtain by the stimulation of each

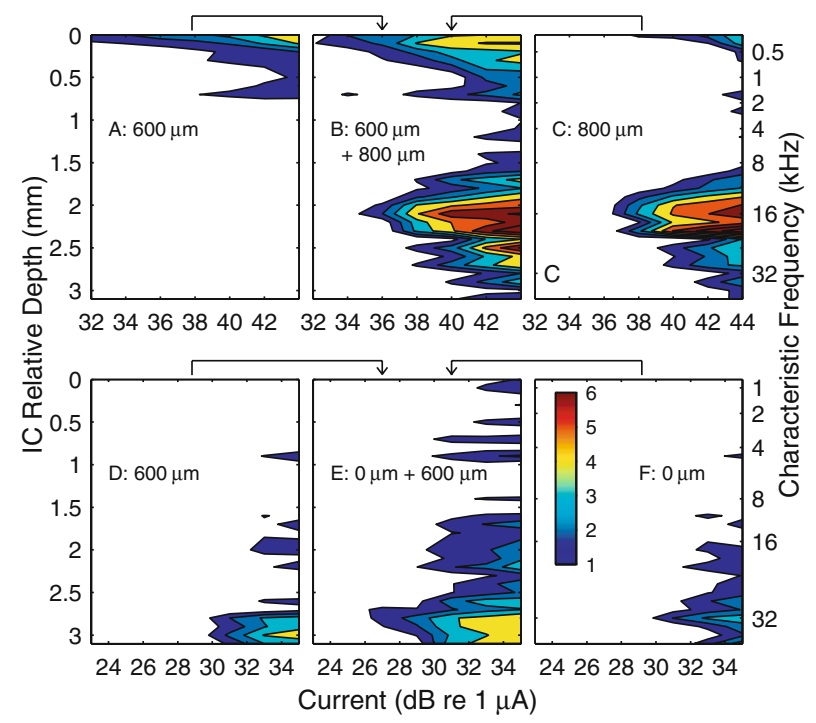

FIG. 9. Simultaneous stimulation of intraneural electrodes. (A), (B), and (C) show an example from Cat0502, in which there was little overlap of spatial tuning curves. In that example, simultaneous stimulation at two sites (B) resulted in minimal reduction of threshold. (D), (E), and (F) show an example from Cat0601, in which there was considerable overlap of spatial tuning curves. In that case, simultaneous stimulation of the two individual sites resulted in a threshold reduction of $\sim 4 \mathrm{~dB}$. 
electrode alone (threshold difference, plotted on the abscissas in Fig. 10) and computed the amount by which the lower of the two single-electrode thresholds was reduced by simultaneous two-electrodestimulation (threshold reduction, plotted on the ordinates). This was done for every ICC recording site at which valid thresholds were available for both single-electrode conditions and for the two-electrode condition (i.e., every condition in which those thresholds fell between the minimum and maximum stimulus levels that were tested). Data were collected for conditions of scala tympani stimulation for a total of 16 stimulating electrode pairs in four cats (one, one, seven, or seven pairs per cat; total of 213 ICC recording sites). For intraneural stimulation conditions, data were collected for a total of 28 stimulating electrode pairs in five cats $(1,3,4,9$, or 11 pairs per cat; total of 293 ICC recoding sites).

Data from the analysis of threshold reductions at individual ICC recording sites are shown in Figure 10 for monopolar scala tympani (Fig. 10A) and intraneural (Fig. 10B) stimulation. Single-electrode threshold differences as large as $\sim 16 \mathrm{~dB}$ were observed for intraneural stimulation, whereas scala tympani threshold differences were $9 \mathrm{~dB}$ or less. The limited range of threshold differences obtained with scala tympani stimulation is consistent with the greater spread of activation observed in that stimulus condition. That is,

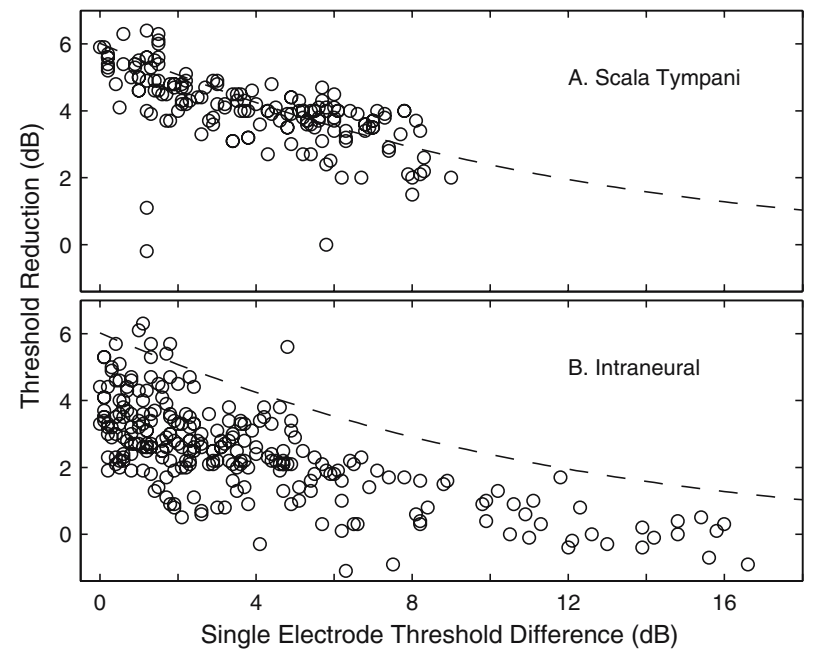

FIG. 10. Threshold reductions at individual ICC recording sites resulting from simultaneous stimulation. Each data point represents the threshold reduction measured at one ICC recording site in response to single- and simultaneous two-electrode stimulation with one pair of electrodes. Single-electrode threshold difference is the difference in the thresholds measured for each electrode alone. Threshold reduction is the threshold for simultaneous two-electrode stimulation subtracted from the lower of the two single-electrode thresholds. The dashed lines indicate the threshold reductions predicted by the model discussed in the text. (A) Monopolar scala tympani stimulation. (B) Intraneural stimulation. at levels $10 \mathrm{~dB}$ above threshold for any given monopolar scala tympani stimulating electrode, essentially all ICC recording sites are active (Fig. 7C), so it is unlikely that a second stimulating electrode would have a threshold more than $9 \mathrm{~dB}$ higher than the first. The number of instances of large (e.g., $>8 \mathrm{~dB}$ ) threshold differences for intraneural stimulation is underrepresented in Figure 10B because, in many cases, ICC recording sites were activated at low threshold by one electrode but could not be activated at any tested level by the second electrode in the pair; in such instances, the threshold difference could not be computed. For that reason, Figure 10B shows a disproportionate number of measurements from the tails of spatial tuning curves. For both scala tympani and intraneural stimulation, there is a general trend of threshold reduction decreasing with increases in the single-electrode threshold difference. Qualitatively, that indicates that currents on two electrodes with nearly equal thresholds contribute equally to a reduced threshold in simultaneous-stimulation conditions and, when two electrodes differ markedly in their individual thresholds, the higher-threshold electrode contributes little to the simultaneous-stimulation threshold. For any particular range of threshold differences, threshold reductions tended to be larger in the scala tympani condition than in the intraneural condition.

The dashed lines in both panels of Figure 10 show the loci of values predicted by a simple model. The model assumes that threshold reductions are a result of electrical current summation among the fibers at the site activated by the lower-threshold stimulating electrode. The single-electrode threshold difference is treated as attenuation in the path to the point of current summation. The dashed lines plot the sum of a unit current plus a second unit current attenuated by the single-electrode threshold difference. Aside from a few outliers, the data points from scala tympani stimulation tend to cluster around the model prediction. In contrast, most of the data from the intraneural stimulation condition lie below the dashed line. The significance of this difference between scala tympani and intraneural stimulating conditions is considered in the "Discussion" section.

\section{Two-pulse temporal integration}

The neural site of excitation in the case of intraneural stimulation is almost certainly the central axons of auditory neurons (i.e., axons of spiral ganglion cells). In contrast, the site of excitation is less certain in the case of scala tympani stimulation and might involve a portion of the central axon, the peripheral processes, and/or the cell body itself. Cartee and colleagues (Cartee et al. 2000, 2006) have argued that differences in the site of excitation 


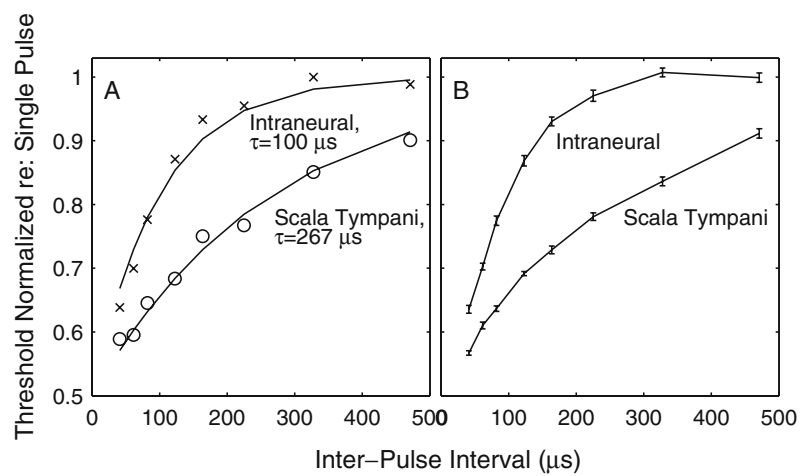

FIG. 11. Two-pulse temporal integration. (A) plots normalized thresholds for two-pulse stimulation as a function of interpulseinterval. Data are from a single ICC recording site tested with intraneural $(X)$ and scala-tympani $(O)$ stimulation. Thresholds were normalized by expressing thresholds in current $(\mu \mathrm{A})$ and dividing each threshold by the threshold for single-pulse stimulation. The solid curves are the best fits of Eq. 1 to the two data sets. (B) shows mean thresholds of all ICC recording sites in one animal at which thresholds could be determined at all tested interpulse intervals. Error bars are standard deviations of the means for 17 ICC sites (intraneural) and 19 sites (scala tympani). The lines simply connect the data points-they are not fit to any particular function. Note that these mean data are shown only to convey the limited variability of the data-all computations of time constants were based on responses at single ICC recording sites. Cat 0513.

should be reflected in the time constants for integration of paired electrical stimulating pulses. Consistent with that argument, they showed that the distribution of time constants differs between those scala tympani and intraneural stimulation sites.

We compared temporal integration time constants for scala tympani and intraneural stimulus conditions in four animals in the present study. Electrical stimuli were pairs of monophasic cathodic pulses, $20 \mu \mathrm{s}$ in phase duration, both presented to the same scala tympani or intraneural electrode. The paired pulses were presented at eight interpulse times, measured from onset to onset, spaced logarithmically from 41 to $471 \mu \mathrm{s}$. Thresholds for activation of ICC units were measured as a function of interpulse time; thresholds were computed using the signal-detection $\left(d^{\prime}\right)$ procedure described in the "Methods" section. The following exponential function (Cartee et al. 2000) was fit to data from each ICC recording site:

$$
\theta_{2 \mathrm{P}}=-0.5 e^{(-\Delta t / \tau)}+1.0
$$

where $\theta_{2 \mathrm{P}}$ is the two-pulse threshold (in $\mu \mathrm{A}$ ) divided by the single-pulse threshold (in $\mu \mathrm{A}$ ), $\Delta t$ is the interpulse interval (in $\mu \mathrm{s}$ ), and $\tau$ is the time constant (in $\mu$ s).

Time constants for two-pulse temporal integration were substantially longer for scala tympani than for intraneural stimulation ( $p<0.001 ; 2$-tailed $t$ test). A plot of normalized threshold versus interpulse-interval for a single ICC recording site is shown in Figure 11A. Each threshold was normalized by expressing it in current $(\mu \mathrm{A})$ and dividing by the threshold for a single pulse. In the figure, Xs and Os represent data for intraneural and scala tympani stimulation, respectively, and the solid lines are the best fits of Eq. 1 . Figure 11B shows the mean thresholds for all ICC recording sites tested in one animal with intraneural (17 recording sites) and monopolar scala tympani (19 recording sites) stimulation. The lines in that figure simply connect the data points-they were not fit to any particular function. Across the four tested animals, time constants from monopolar scala tympani and intraneural stimulation formed discrete unimodal distributions with means and standard deviations of $260 \pm 85 \mu$ s for the scala tympani condition compared to $103 \pm 33 \mu$ s in the intraneural condition (Fig. 12). The illustrated histograms include all the ICC recording sites at which the range of tested stimulus levels permitted threshold determinations at all tested interpulse intervals; the number of sites is larger for scala-tympani stimulation (Fig. 12A) because scala-tympani stimulation produced more widespread activation in the ICC. The significance of those values are considered in the "Discussion" section and compared with those obtained by Cartee and colleagues.

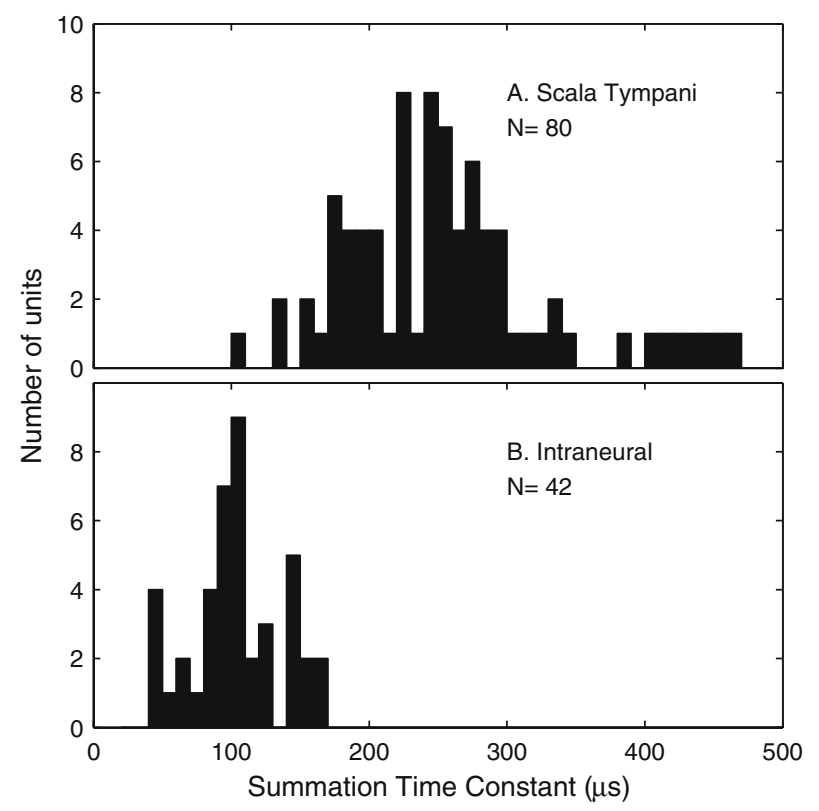

FIG. 12. Distribution of time constants for two-pulse temporal integration. Time constants were computed according to Eq. 1 for individual ICC recording sites in four animals. (A) Monopolar scala tympani stimulation (mean and standard deviation: $260 \pm 85 \mu \mathrm{s}$ ). (B) Intraneural stimulation $(103 \pm 33 \mu \mathrm{s})$. The number of ICC units represented in (A) is larger than that in (B) because the scala tympani stimulus activated a more wide-spread population of ICC neurons. 


\section{DISCUSSION}

The results presented here demonstrate that a penetrating intraneural electrode can activate populations of auditory-nerve fibers with greater specificity, at lower currents, and with less interference among electrodes than is possible with a conventional monopolar scala tympani implant. We begin this discussion by relating the present observations to previous studies of intraneural stimulation of the auditory nerve. Then, we evaluate spread of excitation, thresholds, and range of access to specific frequency representations for intraneural stimulation compared with those measures for scala tympani stimulation. We evaluate between-channel interference for intraneural and scala tympani stimulation. Finally, we consider some of the benefits that might be anticipated if intraneural stimulation were to be employed in an auditory prosthesis system for human use.

\section{Previous studies of intraneural stimulation}

Perceptual effects of intraneural stimulation were evaluated in a small number of human volunteers in pioneering studies by Simmons and colleagues (Simmons 1966; Simmons et al. 1979; Simmons 1983). Those experiments used stimulating arrays consisting of six stainless steel or four platinumiridium wires, $75 \mu \mathrm{m}$ in diameter. The arrays were inserted into the modiolar trunk of the auditory nerve. The subjects were able to distinguish among stimulated electrodes, current levels, and the rates of pulsatile stimulation. Simultaneous stimulation of two electrodes produced no change in threshold, consistent with the present results and in contrast with scala tympani stimulation. Simmons and colleagues also tested intraneural stimulation in cats, using platinum-iridium wire electrodes and monitoring responses by recording electrically evoked auditory brainstem responses (EABR) (Simmons 1979) or cortical surface potentials (Lusted and Simmons 1984). In both the human and animal experiments, thresholds were substantially higher than in the present study: 4 to $20 \mathrm{nC}$ per pulse for perceptual threshold in humans and a median of $5 \mathrm{nC}$ for EABR threshold in cats compared to a mean of $0.8 \mathrm{nC}$ in the present unit recording study in cats. Possible explanations for the higher thresholds in the earlier studies include differences in the locations of the electrodes, polarization of the wire electrodes that were used by the Simmons group, and encapsulation of the wires by reactive tissue.

Two research groups have tested intraneural stimulation using silicon-substrate arrays. Zappia and colleagues (Zappia et al. 1990) tested a five-electrode single-shank thin-film array similar to that used in the present study. They implanted the stimulating array for periods of 5 weeks in guinea pigs, using a lateral approach through the cochlear basal turn. Histological study showed that the implants were tolerated well by the cochlear tissue, with damage in most cases restricted to the loss of spiral-ganglion cells near the implanted silicon shank. Functional responses to biphasic electrical pulses were monitored as middlelatency responses. Thresholds ranged from 1 to $4 \mathrm{nC}$, somewhat higher than that obtained in the present study. Hillman and colleagues (Hillman et al. 2003) tested in cats a silicon-substrate device consisting of 6 to 19 1-mm-long silicon shanks, spaced on a $400-\mu \mathrm{m}$ grid, with one stimulating site at the tip of each shank. The median EABR threshold was $1.1 \mathrm{nC}$.

Cartee and colleagues employed intraneural stimulation in a study of the site of excitation by electrical cochlear stimulation (Cartee et al. 2000; Cartee et al. 2006). They compared time constants for two-pulse temporal integration between scala-tympani and intraneural stimulation sites in the cat. Stimulating electrodes were a single ball in the basal turn of the scala tympani and a sharpened tungsten microelectrode inserted into the modiolar trunk of the auditory nerve. Recordings were made from individual auditory nerve fibers. Time constants for scala tympani stimulation formed a bimodal distribution, with modes lying between $\sim 100$ and $200 \mu$ s and between $\sim 250$ and $350 \mu$ s (Cartee et al. 2006). Time constants for intraneural stimulation formed a unimodal distribution that coincided roughly with the shorter mode of the scala tympani data. Cartee and colleagues concluded that the longer scala tympani time constants probably resulted from the relatively large charge-holding capacity of spiral ganglion cell bodies and probably indicated a site of excitation adjacent to the cell bodies. In contrast, the shorter scala tympani time constants were consistent with excitation of axons central to the cell bodies. In the present study, time constants derived from scala tympani and intraneural stimulation formed largely nonoverlapping unimodal distributions with means of 260 and $103 \mu$ s, respectively, somewhat shorter than those of the two modes observed by Cartee and colleagues (2006). In response to scala tympani stimulation, few units showed short time constants consistent with an axonal site of excitation. The latter observation might reflect the fact that we recorded from ICC neurons that each integrate input from multiple auditory afferents. Similarly, a study in guinea pigs using stimulation with a scala tympani ball electrode and recording from the auditory cortex produced two-pulse time constants ranging from 236 to $294 \mu$ s, consistent with a cell-body site of excitation (Middlebrooks 2004). 
Comparisons of ICC responses to acoustic, scala tympani, and intraneural stimulation

We compared responses at the same ICC sites to acoustic stimulation in normal-hearing conditions with scala tympani and intraneural electrical stimulation in deafened conditions. We inferred the spread of excitation in the auditory nerve by observing the spread of activation at recording sites along the tonotopic axis of the ICC. In the present study, single tone frequencies $>4 \mathrm{kHz}$ presented in the normalhearing condition produced ICC spatial tuning curves that were around $500 \mu \mathrm{m}$ wide at levels $10 \mathrm{~dB}$ above threshold; tuning curves tended to be somewhat wider for lower-frequency tones. Those spatial tuning curve widths for tones are comparable with previous reports in cat ICC (Snyder et al. 1990, 1991; Shepherd et al. 1999).

Electrical stimulation using conventional scala tympani electrode arrays consistently produces greater spread of excitation than that produced by acoustic tones. Near-threshold spatial tuning widths for monopolar scala tympani stimulation were $>1,500 \mu \mathrm{m}$ in the present study using a banded electrode array and an average of $1,250 \mu \mathrm{m}$ wide in a previous study that used a space-filling electrode customized to the cat cochlea. Most previous physiological studies of scala tympani stimulation have employed closely spaced bipolar electrode pairs. In the conditions producing the most restricted spread of excitation-a closely spaced radial or near-radial bipolar pair at the apical end of a space-filling carrier customized for the cat-spatial tuning curve widths were around $700 \mu \mathrm{m}$ at levels $6 \mathrm{~dB}$ above threshold (Snyder et al. 1990; Rebscher et al. 2001). Those spatial tuning curves broadened with increasing stimulus levels, however, so even the narrowest illustrated tuning curves were $\sim 2,000 \mu \mathrm{m}$ in width at $12 \mathrm{~dB}$ above threshold. Widths were appreciably greater for more basal electrode pairs (Snyder et al. 1990) or for electrode arrays that were not custom fitted to the cat.

The spread of activation elicited by intraneural stimulation consistently was more restricted than for monopolar scala tympani stimulation and, in most cases, was more restricted than for bipolar scala tympani stimulation. We observed considerable variation in the spread of excitation resulting from intraneural stimulation, with a few stimulation sites producing widespread or bilobed spatial tuning curves. Nevertheless, in most tested animals, we observed stimulation sites in each of low-, middle-, and high-frequency regions that produced narrow spatial tuning curves, 500 to $1,000 \mu \mathrm{m}$ in width at $6 \mathrm{~dB}$ above threshold. Moreover, intraneural stimulation produced broader dynamic ranges of level over which excitation was restricted to a contiguous frequency range. That is, median values of FSDR were $16.5,>15.5$, and $>12.5 \mathrm{~dB}$ for low-, middle-, and highfrequency regions. Those values are appreciably greater than the median value, $3 \mathrm{~dB}$, of the greatest FSDR obtained in each animal tested with monopolar scala tympani stimulation.

Intraneural stimulation permitted frequency-specific stimulation within frequency ranges spanning a broad range of frequencies. Limitations in the calibrated range of our sound-delivery system $(0.5$ to $40 \mathrm{kHz}$ ) and the finite length of our 32-site recording probes restricted the range of the ICC tonotopic axis that we could sample in each animal. Intraneural stimulation produced examples of restricted spatial tuning curves centered at ICC sites with CFs spanning 0.6 to $38 \mathrm{kHz}$. Other restricted tuning curves appeared to be centered beyond the range of the ICC that we could map with acoustical tones (e.g., Figs. 5D, H; 6A; and 8A). For that reason, we infer that intraneural stimulation sites in 5 of the 10 tested animals stimulated ICC sites with CFs below $0.5 \mathrm{kHz}$ and that stimulation sites in three animals stimulated ICC sites above 32 (two animals) or $40 \mathrm{kHz}$ (one animal). In a study of the map of CF onto the cat's cochlea, Liberman (1982) recorded the CFs of auditory nerve fibers, traced those fibers back to their origins along the organ of Corti, and reported those locations in terms of the percent of the distance from cochlear base to apex. Fibers with CFs as low as $\sim 150 \mathrm{~Hz}$ originated at $\sim 99 \%$ of the distance from the base. Fibers with $\mathrm{CFs}$ around $500 \mathrm{~Hz}$, corresponding to the lowest CFs of our recording and stimulation sites, terminated at $\sim 85 \%$ of the distance from the base. The high-frequency end of Liberman's sample ended at $\sim 35 \mathrm{kHz}$, which mapped onto sites $\sim 5 \%$ of the distance from the base. We observed one stimulation site that produced a spatial tuning curve centered at $38 \mathrm{kHz}$ and others that appeared to peak at CFs beyond 32 or $40 \mathrm{kHz}$. By comparison with Liberman's data, therefore, we infer that our intraneural stimulation sites activated fibers with cochlear terminations ranging from $<5 \%$ to $\sim 85 \%$ of the cochlear distance from base to apex.

In contrast to intraneural arrays, scala tympani electrode arrays in the present study could be positioned only in the basal and a portion of the second cochlear turns, and activation of low-to-midfrequency ICC regions (e.g., $<4 \mathrm{kHz}$ ) by scala tympani stimulation was obtained only when current levels were increased above the threshold for activation of higher-frequency regions. In that condition, presumably, current spread from basal-turn electrodes to activate passing fibers originating in the cochlear apex. In published studies of bipolar scala tympani stimulation in the cat, the lowest-frequency 
spatial tuning curves were located at ICC depths at which neurons typically have CFs of 6-8 kHz (Snyder et al. 1990; Shepherd et al. 1999; Rebscher et al. 2001).

Thresholds for intraneural stimulation were substantially lower than for scala tympani stimulation. In the present study in which scala tympani and intraneural stimulation thresholds were compared at the same ICG recording sites, intraneural thresholds averaged $24.5 \mathrm{~dB}$ lower than monopolar thresholds and $34.1 \mathrm{~dB}$ lower than bipolar thresholds. Bipolar is the more appropriate scala tympani configuration to compare with intraneural stimulation because only the bipolar configuration produces spread of activation that approaches the restricted spread that is produced by intraneural stimulation. The 34.1-dB difference in mean thresholds corresponds to a $\sim 50$ fold lower threshold current for intraneural stimulation. When thresholds are expressed in terms of charge per phase (current multiplied by phase duration), the scala tympani thresholds that were obtained in the present study are comparable to those reported by other groups (Snyder et al. 1990; Shepherd et al. 1999; Rebscher et al. 2001). As noted above, the $\sim 0.8 \mathrm{nC}$ mean threshold obtained for intraneural stimulation in the present study was somewhat lower than that obtained for previous measures of intraneural thresholds in the cat based on recordings of EABR (Simmons 1979; Hillman et al. 2003) and middle-latency response (Zappia et al. 1990).

\section{Interference among multiple electrodes}

As a measure of between-channel interference in the present study, we measured the thresholds for single pulses presented simultaneously on pairs of electrodes compared to the thresholds obtained from stimulation of either electrode alone. We regard a reduction of threshold resulting from simultaneous stimulation as an indication of interference between electrodes. We presume that simultaneous stimulation shows between-channel interference at its worst because, in that condition, neural elements are exposed to the sum of currents on the two electrodes. Indeed, in a condition of monopolar scala tympani stimulation in which there was extensive overlap of stimulus electrical fields, threshold shifts due to simultaneous two-electrode stimulation could be modeled accurately by the simple addition of currents (Middlebrooks 2004), and threshold shifts could be reduced considerably by the introduction of a brief temporal offset between channels (Bierer and Middlebrooks 2004; Middlebrooks 2004). In the present study, we chose to test the worst case because we expected that intraneural stimulation might provide sufficient independence of channels to permit multichannel stimulation with simultaneous continuous waveforms. That expectation was born out in tests of simultaneous two-electrode intraneural stimulation. In cases in which spatial tuning curves elicited by intraneural stimulation showed little overlap, the two spatial tuning curves superimposed in simultaneous-stimulation conditions, preserving the structure of each spatial tuning curve with essentially no reductions in thresholds at the tip of each tuning curve (i.e., averages of $0.3 \mathrm{~dB}$ ). When intraneural spatial tuning curves overlapped substantially, however, threshold reductions at the tips of tuning curves averaged $3.6 \mathrm{~dB}$. Nearly all spatial tuning curves elicited with monopolar scala tympani stimulation showed considerable overlap in the present study, and threshold reductions were large, averaging $4.2 \mathrm{~dB}$.

Analysis of simultaneous-stimulation threshold reductions measured at individual ICC recordings sites (Fig. 10) demonstrates two factors that contribute to greater interference between scala tympani electrodes than between intraneural electrodes. First, the individual stimulation of each of the two intraneural electrodes could show greater threshold differences than was observed for intrascalar stimulation. That follows from the reduced overlap between intraneural spatial tuning curves, due in turn to reduced spread of activation by intraneural stimulation and larger dynamic ranges over which spread of activation was restricted (i.e., larger FSDRs). Second, within any particular range of single-electrode threshold differences, threshold reductions tended to be smaller for intraneural than for intrascalar stimulation. We presume that most or all of the observed threshold reduction for both modes of stimulation is due to the summation of currents within the cochlea or auditory nerve. Threshold reductions for simultaneous scala tympani stimulation cluster around the loci of points predicted by a simple summation model (i.e., the dashed line in Fig. 10A). That suggests that the location of stimulating electrodes in the electrolyte-filled scala tympani affords lowresistance current paths among electrodes. In contrast, threshold reductions resulting from intraneural stimulation (Fig. 10B) tend to be smaller that those predicted by the model, suggesting that the fascicular organization of auditory nerve fibers results in more restricted current paths and more complicated vector sums of currents. We note that it is a goal of presentday cochlear-implant design to create space-filling scala tympani electrode arrays that position electrodes close to the modiolar wall of the scala, thereby restricting current paths within the scala tympani.

The arithmetic model that we evaluated (i.e., represented by the dashed lines in Fig. 10) suggests 
that the simple summation of currents is sufficient to account for the observed threshold reductions resulting from simultaneous stimulation; we regard the points lying above the model prediction in Figure $10 \mathrm{~A}$ as due to the combined variance of the three independent threshold measurements that were required for each data point (i.e., each electrode alone and the simultaneous case). The present experiments cannot rule out an additional contribution of facilitatory or inhibitory interactions within central pathways. For instance, the tendency of data points to lie below the model prediction, particularly in the case of intraneural stimulation, might be a result of the focal activation of inhibitory surrounds in the frequency response areas of auditory brainstem neurons.

In the majority of clinical cochlear prosthesis systems, interference among channels is reduced considerably by interleaving pulses on the various electrodes so that nearby electrodes are never stimulated simultaneously (Wilson et al. 1991). In a study of auditory cortical responses to scala tympani stimulation in guinea pigs, interference between simultaneously presented monopolar pulse trains was essentially eliminated by the introduction of a 2-ms temporal offset between pulses on nearby electrodes (Middlebrooks 2004). Temporal offsets that long would not be practical for multichannel stimulation at the pulse rates that are favored for contemporary speech processors (i.e., $>800 \mathrm{pps}$ ). Two-electrode stimulation using more feasible temporal offsets (i.e., 40 to $120 \mu \mathrm{s}$ ) resulted in threshold shifts of a few decibels. In the present study, intraneural stimulation showed shorter time constants for two-pulse temporal integration than did scala tympani stimulation (Figs. 10 and 11). That suggests that temporal integration between interleaved pulse trains would be reduced in conditions of intraneural stimulation, thereby reducing between-channel interference in interleaved stimulation strategies.

\section{Intraneural stimulation for auditory prosthesis}

The present results obtained in this animal model suggest several benefits that might be obtained if intraneural stimulation were developed for an auditory prosthesis. One obvious application would be cases in which the scala tympani is occluded by bone, precluding the implantation of a scala tympani electrode. Such a condition often occurs as a sequela of meningitis or severe otosclerosis. Another possible benefit would be in cases in which there is residual hearing to be preserved. In principle, an intraneural stimulating array could be inserted into the nerve in the internal meatus without disrupting middle-ear sound conduction or cochlear mechanics and with- out risking additional loss of cochlear hair cells by the introduction of a foreign body into the scala tympani. Even in cases in which a scala-tympani implantation would be routine, however, intraneural stimulation might offer several advantages that derive from more restricted activation of specific neural populations, direct access to fibers from the cochlear apex serving low-frequency hearing, lower thresholds, and reduced interference among multiple stimulus channels.

\section{Restricted spread of excitation}

The present results in an animal model demonstrate more restricted spread of excitation with intraneural stimulation than with monopolar and, perhaps, bipolar scala tympani stimulation; the comparison with bipolar stimulation depends on one's interpretation of the differing dynamic ranges (i.e., differing discrimination slopes). One might predict that more restricted excitation patterns in an auditory prosthesis would provide the more precise transmission of spectral information and, consequently, better speech reception. Contrary to that prediction, comparisons of speech reception among various electrode configurations have failed to demonstrate consistent improvements in speech reception using configurations that produce restricted excitation patterns, such as narrow bipolar or tripolar configurations (Lehnhardt et al. 1992; von Wallenberg et al. 1995; Pfingst et al. 1997; Zwolan et al. 1996; Mens and Berenstein 2005). For that reason, it is an open question whether the restricted excitation patterns produced by intraneural stimulation would provide enhanced speech reception. There are several factors that might account for the poor success in clinical tests of bipolar or tripolar scala tympani stimulation. One factor is that their thresholds are elevated relative to the monopolar configuration, so that stimulators cannot deliver enough current to reach the upper end of dynamic ranges, i.e., maximum comfortable loudness. In contrast, thresholds for intraneural stimulation are substantially lower than for any scala tympani configuration, so it should be no problem to achieve a full range of loudness with intraneural stimulation. Another factor is suggested by our results from physiological studies in guinea pigs. In that animal model, sensitivity to amplitude modulation is markedly decreased in conditions of bipolar compared to monopolar stimulation (Middlebrooks 2005). Impaired modulation sensitivity would have an adverse effect on speech reception and might account for disappointing speech reception results with bipolar (and tripolar) configurations. In ongoing studies, we are assessing the capacity of intraneural stimulation to transmit information about amplitude modulation. 
Contemporary cochlear-implant systems that employ scala-tympani electrode arrays suffer from a limited number of functionally independent electrodes. In speech tests, users of conventional cochlear implants appear to benefit from no more than 4-7 stimulation channels in quiet conditions or 7-10 channels in a noisy background, despite having up to 24 electrodes in their scala tympani arrays (Fishman et al. 1997; Friesen et al. 2001); in parallel tests, normal-hearing subjects listening to a simulation of a cochlear-implant speech processor benefited from more than 20 channels (Friesen et al. 2001). In the present physiological study, stimulation at most intraneural sites produced frequency-specific spread of excitation that was more restricted than that resulting from scala tympani stimulation. Moreover, the dynamic range over which spread of excitation remained spatially restricted consistently was greater for intraneural than for scala tympani stimulation. The reduced spread of excitation afforded by intraneural stimulation resulted in reduced overlap among activated neural populations. When pairs of intraneural electrodes were stimulated simultaneously, the resulting compound spatial tuning curve preserved intact the contribution of each electrode, and each electrode produced negligible interference with the threshold of the other electrode. Those three characteristics of intraneural stimulation-more precise activation of specific neural populations, increased dynamic range over which frequency specificity is maintained, and reduced interference among channels-all would act to enhance the precision of transmission of spectral (i.e., cochlear-place) information from the stimulating array to the brain and would increase the number of functionally independent channels. Enhanced transmission of spectral information and increased functional channel count in a prosthesis presumably would enhance recognition of speech, musical timbre, and other sounds and would enhance the segregation of signals from noisy backgrounds.

The majority of present-day cochlear-implant systems use pulsatile stimulation schemes intended to reduce interference among channels. In such schemes, electrical stimuli consist of constant-rate pulse trains presented in a temporally interleaved fashion to multiple scala tympani electrodes. Sound waveforms are processed by a bank of bandpass filters, the amplitude envelope is extracted from each frequencyspecific channel, and the envelope from each channel is used to amplitude-modulate the pulse train delivered to a particular intracochlear electrode (Wilson et al. 1991). The natural amplitude envelopes of sounds are preserved in such processing schemes, but acoustic fine structure is eliminated, being replaced by the constant-rate electrical pulse train. A psychophysical study that put envelope information in opposition with fine-structure information demonstrated that the envelope of a sound waveform dominates speech recognition and that fine structure is most important for pitch perception and sound localization based on interaural time differences (ITD) (Smith et al. 2002). The much-reduced levels of between-channel interference observed with intraneural stimulation raises the possibility of employing electrical stimulation with continuous analog waveforms that would preserve the natural fine structure of acoustic signals. One might expect the preservation of the fine structure of sounds to enhance pitch perception and, in binaural applications, enhance directional hearing based in ITDs.

\section{Access to fibers from the apical cochlea}

In the present study, intraneural stimulation permitted frequency-specific stimulation of fibers spanning most of the cat's cochlear frequency representation, including low-frequency fibers with CFs below $500 \mathrm{~Hz}$ originating $\sim 85 \%$ of the cat's cochlear length from the cochlear base; in humans, the $85 \%$ locus is estimated to represent a frequency of $\sim 175 \mathrm{~Hz}$ (Greenwood 1990). We presume that the demonstration of access to even lower CFs was limited in the present study primarily by the range of the ICC tonotopic map that we could sample. We routinely placed the recording probe to sample ICC neurons tuned to $32 \mathrm{kHz}$ or higher. Given the 3.1-mm span of recording sites on the probe, therefore, the lowfrequency end of the tonotopic axis that we could sample was $\sim 500 \mathrm{~Hz}$. Access to the cochlear apex with scala tympani electrodes always will be limited by the depth to which the scala tympani array can be inserted, whereas, in principle, access to apical fibers with an intraneural penetrating array is limited only by the ability to locate those fibers. The selective activation of sites throughout the entire cochlea would further increase the number of functionally independent channels of stimulation to the brain (in addition to the three factors mentioned above in regard to spread of excitation). Moreover, specific stimulation of fibers from the cochlear apex might enhance low-frequency hearing. Scala tympani electrode arrays in clinical use are inserted in the basal (i.e., high-frequency) turn of the cochlea and terminate in the middle turn at loci representing frequencies no lower than about $600 \mathrm{~Hz}$ (Skinner et al. 2002; Wardrop et al. 2005); recent data suggest that some arrays can reach as far apical as the representation of frequency around $300 \mathrm{~Hz}$ (Baumann and Nobbe 2006; Boex et al. 2006). Speech processing programs typically assign low frequencies (i.e., lower than $\sim 200 \mathrm{~Hz})$ to the most apical scala tympani 
electrode, but that creates a mismatch between the normal frequency sensitivity of that cochlear site and the assigned acoustic frequencies that lead to the stimulation of that site.

Frequencies below $600 \mathrm{~Hz}$ include the fundamental (i.e., pitch) frequencies of the human voice (typically averaging 100, 200, and $250 \mathrm{~Hz}$ for men, women, and children, respectively) and the pitch frequencies of most musical melodies; for instance, $600 \mathrm{~Hz}$ is $\sim 11 / 4$ octaves above middle $\mathrm{C}$ on the piano. Cochlear-implant users typically show poor pitch discrimination and very limited ability to recognize musical melodies (McDermott 2004; Pressnitzer et al. 2005; Vandali et al. 2005). Pitch sensation in cochlear-implant users is influenced by the tonotopic locations of stimulating electrodes ("place pitch") and by stimulation rate ("rate pitch"). A recent study using deep scala tympani insertions reported place pitch sensations as low as around $300 \mathrm{~Hz}$, but most of those experimental subjects reported the same pitch resulting from the two most apical electrodes located $2 \mathrm{~mm}$ apart (Baumann and Nobbe, 2006), which would span nearly an octave in estimates of the cochlear frequency map (Greenwood 1990). Normalhearing listeners can recognize pitches on the basis of periodicity cues even in cases in which no energy is present at the fundamental; for instance, one can recognize the pitch of a man's voice over a telephone although the telephone does not transmit low frequencies. Such recognition of periodicity pitch requires information about the fine structure of acoustical waveforms, however, and that fine structure is eliminated by the pulsatile processing schemes that are used in the majority of cochlear-implant systems (Wilson et al. 1991). Under normal hearing conditions, the fundamental periodicity is present in the envelopes of high-frequency auditory filters, but the modulation waveforms used in most cochlear implants are low-pass filtered below the relevant pitch frequencies. Implant users report a rate pitch sensation that varies with the carrier rate of a pulse train, but sensitivity to pulse rate is largely lost at rates above $300 \mathrm{~Hz}$ (Shannon 1983; Zeng 2002). Moreover, contemporary cochlear-implant systems use a constant pulse rate for all channels irrespective of the acoustic input. We anticipate that intraneural stimulation could enhance low-frequency hearing by providing frequency-specific activation of the most apical cochlear fibers.

Another possible benefit of the ability to stimulate low-frequency fibers from the cochlear apex is that, in a binaural application, specific apical stimulation might enhance spatial hearing. The dominant cue for sound localization in normalhearing listeners is the ITD in the fine structure of sounds at frequencies below $\sim 1.5 \mathrm{kHz}$ (Wightman and Kistler 1992; Macpherson and Middlebrooks 2002). Thresholds for detection of ITD in lowfrequency sounds can be as low as $\sim 20 \mu$ s (Licklider et al. 1950; Zwislocki and Feldman 1956; Yost et al. 1971). Thresholds for fine-structure delays are processed by a pathway through the medial superior olivary nucleus (MSO; (Goldberg and Brown 1969). At least in experimental animals, the frequency representation in the MSO is heavily biased to low frequencies (Osen 1969; Guinan et al. 1972), suggesting that the processing of interaural fine-structure delays is an apical cochlear function. Normal-hearing listeners also can detect interaural delays in the envelopes of high-frequency sounds. Under certain laboratory conditions, thresholds for the detection of envelope delays can be as low as those for fine structure delays (Yost 1976; Bernstein and Trahiotis 2002), but in most conditions, envelope delay thresholds are considerably higher, $>100 \mu$ s (Yost et al. 1971; Nuetzel and Hafter 1981; Bernstein and Trahiotis 1994). Moreover, ITDs in most high-frequency sounds have little influence on the perceived laterality of a sound (Bernstein and Trahiotis 1985; Macpherson and Middlebrooks 2002). Tests of interaural delay detection by binaural cochlear-implant users under conditions in which the fine timing of pulse trains was controlled at both ears have demonstrated thresholds as high, or higher, than those for the detection of high-frequency envelope delays by normal-hearing listeners (van Hoesel and Tyler 2003; Majdak et al. 2006). We speculate that contemporary cochlear implants do not allow full access to the MSO mechanisms that are specialized for detecting interaural fine-structure delays. Direct stimulation of apical fibers using intraneural stimuli might engage low-frequency interaural delay pathways that could enhance spatial hearing by bilateral auditory prosthesis users.

\section{Reduced current requirements}

Conventional cochlear-implant systems require behind-the-ear battery packs and, typically, daily battery replacement. At least part of that electrical power consumption reflects the relatively high currents needed for auditory nerve excitation due to the remote position of scala tympani electrodes relative to auditory nerve fibers. In the present study, thresholds for intraneural stimulation were $\sim 16$-fold less than those for monopolar and $\sim 50$-fold less than those for bipolar scala tympani stimulation. That difference presumably would result in a substantial reduction in power consumption in a practical clinical device and, indeed, might lead to a totally implantable device not needing an external battery pack. 


\section{CONCLUDING REMARKS}

The present study demonstrates specific activation of the auditory pathway by the stimulation of the auditory nerve with a penetrating multielectrode array and suggests several benefits that might be realized if intraneural stimulation were incorporated into an auditory prosthesis for deaf people. Of course, numerous challenges must be overcome before one could even consider such an application. Among these are engineering issues pertaining to the design of the actual intraneural electrode array and associated stimulating hardware; surgical and medical issues pertaining to device implantation, longterm tolerance of the electrode array by neural tissue, etc.; and signal processing issues related to sound processing strategies that would exploit all the potential advantages of intraneural stimulation. Some issues addressable in an animal model include the problem of "unwrapping" the nonmonotonic map of frequency across depth in the auditory nerve and characteristics of intraneural responses to pulsatile versus continuous waveforms. Our continuing studies are addressing these issues and others, motivated by the goal of realizing intraneural stimulation as a practical mode of auditory prosthesis.

\section{ACKNOWLEDGEMENTS}

We thank James Wiler for expert technical assistance; Zekiye Onsan for help with the illustrations; and Dr. Jochen Schacht, Dr. Bryan Pfingst, Dr. Ewan Macpherson, and the two anonymous reviewers for their helpful suggestions on the manuscript. This work was supported by National Institute on Deafness and Other Communication Disorders contract NO1-DC5-0005 and grant P30 DC05188.

\section{REFERENCES}

Arnesen AR, Osen KK. The cochlear nerve in the cat: topography, cochleotopy, and fiber spectrum. J. Comp. Neur. 178:661-678, 1978.

Arts HA, Jones DA, Anderson DJ. Prosthetic stimulation of the auditory system with intraneural electrodes. Ann. Otol. Rhinol. Laryngol. 112(Suppl 191):20-25, 2003.

Baumann U, Nobbe A. The cochlear implant electrode-pitch function. Hear. Res. 213:34-42, 2006.

BernsteIn LR, TRAHIOTIS C. Lateralization of sinusoidally amplitudemodulated tones: effects of spectral locus and temporal variation. J. Acoust. Soc. Am. 78:514-523, 1985.

Bernstein LR, Trahiotis C. Detection of interaural delay in highfrequency sinusoidally amplitude-modulated tones, two-tone complexes, and bands of noise. J. Acoust. Soc. Am. 95:35613567, 1994.

Bernstein LR, Trahiotis C. Enhancing sensitivity to interaural delays at high frequencies by using "transposed stimuli." J. Acoust. Soc. Am. 112:1026-1036, 2002.
Bierer JA, Middlebrooks JC. Auditory cortical images of cochlearimplant stimuli: dependence on electrode configuration. J. Neurophysiol. 87:478-492, 2002.

Bierer JA, Middlebrooks JC. Cortical responses to cochlear implant stimulation: channel interactions. J. Assoc. Res. Otolaryngol. 5:32-48, 2004.

Boex C, Baud L, Cosendai G, Sigrist A, Kos M-I, Pelizzone M. Acoustic to electric pitch comparisons in cochlear implant subjects with residual hearing. J. Assoc. Res. Otolaryngol. 7:110 124, 2006

Cartee LA, van den Honert C, Finley CC, Miller RL. Evaluation of a model of the cochlear neural membrane. I. Physiological measurement of membrane characteristics in response to intrameatal electrical stimulation. Hear. Res. 146:143-152, 2000.

Cartee LA, Miller CA, van den Honert C. Spiral ganglion cell site of excitation I: comparison of scala tympani and intrameatal electrode responses. Hear. Res. 215:10-21, 2006.

Fishman KE, Shannon RV, Slattery WH. Speech recognition as a function of the number of electrodes used in the SPEAK cochlear implant speech processor. J. Speech Lang. Hear. Res. 40:1201-1215, 1997.

Friesen LM, SHANNON RV, BASkent D, WANG X. Speech recognition in noise as a function of the number of spectral channels: comparison of acoustic hearing and cochlear implants. J. Acoust. Soc. Am. 110:1150-1163, 2001.

Goldberg JM, Brown PB. Response of binaural neurons of dog superior olivary complex to dichotic tonal stimuli: some physiological mechanisms of sound localization. J. Neurophysiol. 32:613-636, 1969.

Green DM, Swets JA. Signal Detection Theory and Psychophysics. New York, Wiley, 1966.

GREENWOOD DD. A cochlear frequency-position function for several species-29 years later. J. Acoust. Soc. Am. 87:2592-2605, 1990.

Guinan JJ JR, Norris BE, Guinan SS. Single auditory units in the superior olivary complex II: locations of unit categories and tonotopic organization. Int. J. Neurosci. 4:147-166, 1972.

Hillman T, Badi AN, Normann RA, Kertesz T, Shelton C. Cochlear nerve stimulation with a 3-dimensional penetrating electrode array. Otol. Neurotol. 24:764-768, 2003.

Lehnhardt E, Gnadeberg D, Battmer RD, von Wallenberg E. Experience with the cochlear miniature speech processor in adults and children together with a comparison of unipolar and bipolar modes. ORL J. Otorhinolaryngol. Relat. Spec. 54:308313, 1992

Liberman MC. The cochlear frequency map for the cat: labeling auditory-nerve fibers of known characteristic frequency. $\mathrm{J}$. Acoust. Soc. Am. 72:1441-1449, 1982.

Licklider JCR, Webster JC, Hedlun JM. On the frequency limits of binaural beats. J. Acoust. Soc. Am. 22:468-473, 1950.

Lusted HS, Simmons FB. Interaction of cortical evoked potentials to electric and acoustic stimuli. J. Acoust. Soc. Am. 76:449-455, 1984.

Macmillan NA, Creelman CD. Detection Theory: A User's Guide. Mahwah, Elrbaum, 2005.

Macpherson EA, Middlebrooks JC. Listener weighting of cues for lateral angle: the duplex theory of sound localization revisited. J. Acoust. Soc. Am. 111:2219-2236, 2002.

Majdak P, Laback B, Baumgartner W-D. Effects of interaural time differences in fine structure and envelope on lateral discrimination in electric hearing. J. Acoust. Soc. Am. 120:2190-2201, 2006.

McDermott HJ. Music perception with cochlear implants: a review. Trends Amplif. 8:49-82, 2004.

Mens LHM, Berenstein CK. Speech perception with mono- and quadrupolar electrode configurations: a crossover study. Otol. Neurotol. 26:957-964, 2005. 
Middlebrooks JC. Effects of cochlear-implant pulse rate and interchannel timing on channel interactions and thresholds. J. Acoust. Soc. Am. 116:452-468, 2004.

Middlebrooks JC. Transmission of temporal information from a cochlear implant to the auditory cortex. Abstr. Assoc. Res. Otolaryngol. 28: Program \#584, 2005.

Nuetzel JM, Hafter ER. Discrimination of interaural delays in complex waveforms: spectral effects. J. Acoust. Soc. Am. 69:1112-1118, 1981.

Nuttall AL, Marques DM, Lawrence M. Effects of perilymphatic perfusion with neomycin on the cochlear microphonic potential in the guinea pig. Acta Otolaryngol. 83:393-400, 1977.

OsEN KK. The intrinsic organization of the cochlear nuclei in the cat. Acta Otolarygol. 67:352-359, 1969.

Pfingst BE, Zwolan TA, Holloway LA. Effects of stimulus configuration on psychophysical operating levels and on speech recognition with cochlear implants. Hear. Res. 112:247-260, 1997.

Pressnitzer D, Bestel J, Fraysse B. Music to electric ears: pitch and timbre perception by cochlear implant patients. Ann. N.Y. Acad. Sci. 1060:343-345, 2005.

Rebscher SJ, SNyder RL, LeAKe PA. The effect of electrode configuration and duration of deafness on threshold and selectivity of responses to intracochlear electrical stimulation. J. Acoust. Soc. Am. 109:2035-2048, 2001.

Rose J, Greenwood, DD, Goldberg, JM, Hind, JE. Some discharge characteristics of single neurons in the inferior colliculus of the cat. I. Tonotopical organization, relation of spike-counts to tone intensity, and firing patterns of single elements. J. Neurophysiol. 26:294-320, 1963.

SHANNON RV. Multichannel electrical stimulation of the auditory nerve in man. I. Basic psychophysics. Hear. Res. 11:157-189, 1983.

ShePherd RK, BAXI JH, Hardie NA. Response of inferior colliculus neurons to electrical stimulation of the auditory nerve in neonatally deafened cats. J. Neurophysiol. 82:1363-1380, 1999.

Simmons FB. Electrical stimulation of the auditory nerve in cats: long term electrophysiological and histological results. Ann. Otol. Rhinol. Laryngol. 88:533-539, 1979.

Simmons FB. Electrical stimulation of the auditory nerve in man. Arch. Otorhinolaryngol. 84:24-76, 1966.

Simmons FB. Percepts from modiolar (eighth nerve) stimulation. Ann. N.Y. Acad. Sci. 405:259-263, 1983.

Simmons FB, Epley JM, Lummis RC, Guttman N, Frishkopf LS, Harmon LD, Zwicker E. Auditory nerve: electrical stimulation in man. Science 148:104-106, 1965.

Simmons FB, Mathews RG, Walker MG, White RL. A functioning multichannel auditory nerve stimulator. Acta Otolaryngol. 87:170-175, 1979.

Simmons FB, Mongeon CJ, Lewis WR, Huntington DA. Electrical stimulation of the acoustical nerve and inferior colliculus: results in man. Arch. Otolaryngol. 79:67, 1964.

Skinner MW, Ketten D, Holden LK, Harding GW, Smith PG, Gates GA, Neely JG, Kletzker GR, Brunsden B, Blocker B. CT-Derived estimation of cochlear morphology and electrode array position in relation to word recognition in Nucleus-22 recipients. J. Assoc. Res. Otolaryngol. 3:332-350, 2002.

Smith ZM, Delgutte B, Oxenham AJ. Chimaeric sounds reveal dichotomies in auditory perception. Nature 416:87-90, 2002.

Snyder RL, Bierer JA, Middlebrooks JC. Topographic spread of inferior colliculus activation in response to acoustic and intracochlear electrical stimulation. J. Assoc. Res. Otolaryngol. 5:305-322, 2004.

Snyder RL, Rebscher SJ, Cao K, Leake PA, Kelly K. Chronic intracochlear electrical stimulation in the neonatally deafened cat. I: expansion of central representation. Hear. Res. 50:7-34, 1990.

Snyder RL, Rebscher SJ, Leake PA, Kelly K, CaO K. Chronic intracochlear electrical stimulation in the neonatally deafened cat. II. Temporal properties of neurons in the inferior colliculus. Hear. Res. 56:246-264, 1991.

VAN Hoesel RJM, Tyler RS. Speech perception, localization, and lateralization with bilateral cochlear implants. J. Acoust. Soc. Am. 113:1617-1630, 2003.

Vandali AE, Sucher C, Tsang DJ, McKay CM, Chew JW, McDermott HJ. Pitch ranking ability of cochlear implant recipients: a comparison of sound-processing strategies. J. Acoust. Soc. Am. 117:3126-3138, 2005.

von Wallenberg EL, Battmer R-D, Doden I, Gnadeberg MS, Houtle K, Lenarz T. Place-pitch and speech perception measures with bipolar and monopolar electrical stimulation of the cochlea. Ann. Otol. Rhinol. Laryngol. 104(Suppl 166):372-375, 1995.

Wardrop P, Whinney D, Rebscher S, Luxford W, Leake P. A temporal bone study of insertion trauma and intracochlear position of cochlear implant electrodes. II. Comparison of spiral Clarion and HiFocus II electrodes. Hear. Res. 203:68-79, 2005 .

Wightman FL, Kistler DJ. The dominant role of low-frequency interaural time differences in sound localization. J. Acoust. Soc. Am. 91:1648-1661, 1992.

Wilson BS, Finley CC, Lawson DT, Wolford RD, Eddington DK, RABinowitz WM. Better speech recognition with cochlear implants. Nature 352:236-238, 1991.

Yost WA. Lateralization of repeated filtered transients. J. Acoust. Soc. Am. 60:178-181, 1976.

Yost WA, Wightman FL, GReEn DM. Lateralization of filtered clicks. J. Acoust. Soc. Am. 50:1526-1531, 1971.

Zappia JJ, Hetke JF, Altschuler RA, Niparko JK. Evaluation of a silicon-substrate modiolar eighth nerve implant in a guinea pig. Otolaryngol. Head Neck Surg. 103:575-582, 1990.

ZENG FG. Temporal pitch in electric hearing. Hear. Res. 174:101106, 2002.

Zwislocki J, Feldman RS. Just noticeable differences in dichotic phase. J. Acoust. Soc. Am. 28:860-864, 1956.

Zwolan TA, Kileny PR, Ashbaugh C, Telian SA. Patient performance with the cochlear corporation " $20+2$ " implant: bipolar versus monopolar activation. Am. J. Otol. 17:717-723, 1996. 